\title{
Counter-hairpin vortices in the turbulent wake of a sharp trailing edge
}

\author{
Sina Ghaemi $†$ and Fulvio Scarano \\ Department of Aerodynamics, Delft University of Technology, Kluyverweg 1, \\ 2629 HS, Delft, The Netherlands
}

(Received 12 April 2011; revised 21 September 2011; accepted 29 September 2011)

The unsteady organization and evolution of coherent structures within the turbulent boundary layer and subsequent wake of the sharp symmetric trailing edge of a NACA0012 aerofoil are investigated. The experiments are conducted in an open testsection wind tunnel at $\operatorname{Re}_{c}=386000$ based on the aerofoil chord and $\operatorname{Re}_{\theta}=1300$ based on the boundary layer momentum thickness. An initial characterization of the flow field using two-component particle image velocimetry (PIV) is followed by the investigation of the unsteady organization and evolution of coherent structures by time-resolved three-dimensional PIV based on a tomographic approach (Tomo-PIV). The inspection of the turbulent boundary layer prior to the trailing edge in the region between 0.15 and $0.8 \delta_{99}$ demonstrated streaks of low- and high-speed flow, while the low-speed streaks are observed to be more coherent along with strong interaction with hairpin-type vortical structures similar to a turbulent boundary layer at zero pressure gradient. The wake region demonstrated gradual deterioration of both the low- and the high-speed streaks with downstream progress. However, the low-speed streaks are observed to lose their coherence at a faster rate relative to the high-speed streaks as the turbulent flow develops towards the far wake. The weakening of the low-speed streaks is due to the disappearance of the viscous sublayer after the trailing edge and gradual mixing through the transport of the remaining low-speed flow towards the free stream. This transport of low-speed flow is performed by the ejection events induced by the hairpin vortices as they also persist into the developing wake. The higher persistence of the high-speed streaks is associated with counter-hairpin vortical activities as they oppose the deterioration of the high-speed streaks by frequently sweeping the high-speed flow towards the wake centreline. These vortical structures are regarded as counter-hairpin vortices as they exhibit opposite characteristics relative to the hairpin vortices of a turbulent boundary layer. They are topologically similar to the hairpins as they appear to be U-shaped but with inverted orientation, as the spanwise portion is in the vicinity of the wake centreline and the legs are inclined at an approximately $60^{\circ}$ to the wake axis in the downstream direction demonstrating a strain-dominated topology. The counter-hairpin vortices are partially wrapped around the high-speed streaks and contribute to the wake development by transporting highspeed flow towards the wake centreline. Similar to the hairpin vortices of a turbulent boundary layer, the occurrence of a complete counter-hairpin vortex is occasional while its derivatives (portions of spanwise or quasi-streamwise vortices) are more frequently observed. Therefore, a pattern recognition algorithm is applied to establish characterization based on an ensemble-averaged counter-hairpin vortex. The formation of the counter-hairpin vortices is due to an additional degree of interaction between

$†$ Email address for correspondence: s.ghaemi@tudelft.nl 
the low- and high-speed streaks after the trailing edge across the wake centreline. The shear layer produced along the wake centreline by neighbouring low- and high-speed streaks promotes the formation of spanwise vortices that form the counter-hairpin vortices by connection to quasi-streamwise vortices. Finally, a conceptual model is proposed to depict the three-dimensional unsteady organization and evolution of coherent structures in the wake region based on the hairpin and counter-hairpin vortex signatures.

Key words: boundary layer structure, free shear layers, turbulent boundary layers

\section{Introduction}

Turbulent flow around the trailing edge is considered thoroughly in the design of the wings, high-lift devices and engine fans of aircraft. The fluctuations within the turbulent flow of the trailing-edge wake affect the pressure distribution around the aerofoil and result in unsteady loads and structural vibrations (Sharland 1964; Hebbar 1986). The high-lift devices of the aircraft wing are influenced by the trailing-edge design, as they interact with the wake of the main aerofoil element (Tummers 1999). It is also known that the prominent problem of trailing-edge noise, which includes the noise of the wing, high-lift devices, blades and the outlet guide vanes of the aircraft engine (Brouwer \& Rienstra 2008), is due to the acoustic noise scattering of the turbulent fluctuations in the vicinity of the trailing edge (Ffowcs Williams \& Hall 1970).

The trailing-edge noise can consist of both tonal and broadband emissions (Brooks \& Hodgson 1981). The tonal contribution is due to the vortex shedding by a separated boundary layer or a blunt trailing edge (Brooks, Pope \& Marcolini 1989) or is caused by a self-excited acoustic feedback loop in the case of a laminar boundary layer (Tam 1974; Nash, Lowson \& McAlpine 1999). The fluid mechanic aspects of the vortex shedding phenomenon and suppression methods through aerodynamic design principles and optimization algorithms have been extensively covered in the related literature (Wygnanski, Champagne \& Marsali 1986; Williamson 1996; Marsden et al. 2007). On the other hand, the broadband noise is associated with a non-periodic wake and is dominant at high Reynolds numbers, which are more prevalent for large transport aircraft (Nash et al. 1999). It is generated over a wide range of frequencies as a result of acoustic scattering emanating from the sudden distortion of vortical structures of different length scales (Sandberg \& Sandham 2008; Finez et al. 2010). The rapid distortion of vortices is in response to the abrupt pressure release and the non-equilibrium state immediately downstream of the trailing edge as a result of the geometric truncation. The contributing turbulent aspects are complex and linked to the turbulent boundary layer upstream of the trailing edge.

The unsteady organization of a turbulent boundary layer is modelled by the combination of a set of coherent structures known as the hairpin vortex signature (Adrian, Meinhart \& Tomkins 2000). The vortical structures within this model are the hairpin vortices and the accompanying quasi-streamwise vortices that induce a focused ejection of the viscously retarded flow away from the wall vicinity (Blackwelder \& Eckelmann 1979; Head \& Bandyopadhyay 1981). The ejection events are the major contributor to the Reynolds shear stress above $y^{+}=12$ (Wallace, Eckelmann $\&$ Brodkey 1972). They lift the low-speed streaks and vorticity away from the wall 
and sustain the turbulence production (Kline et al. 1967). In the outboard region of the hairpin vortices, an unfocused induction forms a downward sweep event above the high-speed streaks (Adrian \& Liu 2002) transporting high-speed fluid towards the wall, enhancing drag and heat transfer (Schoppa \& Hussain 2000). In a trailing-edge scenario, this complex mechanism of generation and transport of turbulence in the boundary layer should adapt to the boundary-free condition of the wake region. This evolution of the coherent structures of the turbulent boundary layer into an isotropic state is of fundamental importance, and some aspects of it have been tackled by Hamelin \& Alving (1996) and Morris \& Foss (2003).

The genesis of the hairpin vortices of a turbulent boundary layer either through the instability of the low-speed streaks (Schoppa \& Hussain 2000) or an induction mechanism of an already existing vortex (Zhou et al. 1999) has been a long-standing question. Investigation of the immediate wake region also offers the possibility to study the evolution of the hairpin structures after the disappearance of the solid wall and the viscous sublayer. Formation of new vortical structures in the immediate wake region is also possible due to the new boundary condition. The immediate wake region is distinguished from the upstream boundary layer as the proposed models for wall-bounded flows are not adequate for this non-equilibrium region, and as a result the turbulent wake has drawn the attention of a different body of literature.

The early literature on the wake of a streamlined non-lifting body starts with Chevray \& Kovasznay (1969), who explored the turbulent wake of a thin flat plate using hot-wire anemometry and verified the absence of the large-scale periodic structures often observed in the wake of two-dimensional blunt bodies and mixing layers. Andreopoulos \& Bradshaw (1980) applied temperature-conditioned sampling and observed a region of small-scale mixing in the vicinity of the wake centreline splitting two layers of unmixed fluid. This small-scale mixing is in contrast to the time-sharing process of large eddies occurring in interacting shear layers within ducts and jets (Dean \& Bradshaw 1976). Ramaprian, Patel \& Sastry (1982) conducted hotwire measurements in the wake of a smooth flat plate and statistically characterized different regions of the wake. Hot-wire measurements of Hebbar (1986) showed an overshoot in the streamwise profiles of mean-flow parameters and in the peak values of Reynolds stresses in the immediate wake of a symmetric aerofoil. Hayakawa \& Iida (1992) ascribed this overshoot in turbulence intensity to direct interaction of intense wall turbulence emerging from both sides of the flat plate. Bogucz \& Walker (1988) analysed the wake flow at a sharp trailing edge in the limit of infinite Reynolds number and provided an analytical representation of the experimental data in the near wake. To the authors' knowledge, only Haji-Haidari \& Smith (1988) have visualized the flow structure of the near wake of a flat plate, using a hydrogen bubble technique, and identified instantaneous stretching of streamwise vortices and their organization. They speculated that streamwise vortices along with inrush of fluid from both sides of the plate play a major role in the mixing process. Nakayama \& Liu (1990) studied the effect of variation in Reynolds number, and concluded that the larger eddies of the outer layer modulate the small eddies of the centreline region. These studies have explored the statistics of the turbulent fluctuations and the relative scale of the vortical structures, but the type of the coherent structures causing the small-scale mixing is unclear. This is partially due to the repeated use of a single-point measurement technique (hot-wire anemometer).

The turbulent wake of a symmetric sharp trailing edge is classified into several regions as illustrated in figure 1 . The inner layer is a region of mixing along the centreline in between the two boundary layers, which initiates at the trailing edge 
and widens as it progresses downstream. The vortical structures within the inner layer perform the mixing process and have been referred to as 'fine scale' by Andreopoulos \& Bradshaw (1980). Haji-Haidari \& Smith (1988) reported dominantly streamwise structures associated with an inward motion of fluid towards the wake centreline to be active within this region. These fine-scale vortical structures rapidly increase the velocity of the inner layer profile beyond that of the initial boundary layer and promote the recovery of the wake deficit; however, their kinematics and dynamics are still unclear. The instantaneous boundaries of the inner wake are highly sinuous in shape, resulting in a $14 \%$ probability of the unmixed fluid passing across the wake centreline (Andreopoulos \& Bradshaw 1980). The outer layer is initially unperturbed by the disappearance of the wall and remains similar to the initial boundary layer profile till about $25 \theta_{0}$ ( $\theta_{0}$ is momentum thickness) downstream of the trailing edge according to Ramaprian et al. (1982) or $270 \lambda^{+}\left(\lambda^{+}\right.$is the wall unit) according to Haji-Haidari \& Smith (1988). The reported limits depend on the Reynolds number; however, beyond those the mean velocity in the outer layer begins to reduce and the velocity profile gradually widens. This outer layer mixing is performed by intermittency between the mixed and unmixed fluid rather than the fine-scale structures as described by Andreopoulos \& Bradshaw (1980).

The classification of the wake along the centreline starts with the near wake region where small-scale mixing occurs in the inner layer while the outer layer is still unperturbed and consists of unmixed fluid until a downstream location of $x^{+} \sim 270$ (Haji-Haidari \& Smith 1988). The near wake is characterized by the fastest growth rate of the wake width and the fastest decay of the velocity defect. The initial region of the near wake $\left(0<x^{+}<15\right)$ is affected by the trailing-edge thickness and the viscous-sublayer interaction, encompassing a small unsteady bubble of stagnant flow. Consequently, small counter-rotating vortical structures are formed, which are reported to be similar to those seen in a Kármán vortex street (Miau \& Karlsson 1987). This vortex shedding process exhibits highly viscous behaviour and results in an initial linear increase of the time-averaged centreline velocity similar to the velocity trend in the sublayer of a turbulent boundary layer. An immediate change in the flow parameters such as momentum thickness and friction velocity is also reported (Ramaprian et al. 1982). The shedding process is damped rapidly and the rest of the near wake $15<x^{+}<270$ is non-periodic and dominated by the fine-scale activities of the inner layer (Haji-Haidari \& Smith 1988). In this region, the initial linear trend of the wake centreline velocity undergoes a transitional region similar to the buffer layer of a turbulent boundary layer. The change in the flow structures from the initial viscosity-dominated behaviour to the turbulence-dominated mixing in the rest of the near wake can be investigated and compared to that of a turbulent boundary layer.

The intermediate wake region initiates once the fine-scale mixing of the near wake has spread beyond the inner layers of the two initial boundary layer profiles. In this region, the large eddies in the outer parts of the two boundary layers start to take part in the wake development process, resulting in a gradual exchange of the innerlayer scales to the outer-layer parameters such as wake half-width. The modulation of the small eddies of the centreline region by the larger eddies of the outer layer observed by Nakayama \& Liu (1990) can be a part of this conversion process, so that its detailed mechanism requires further investigation. The mixed-fluid contribution within the intermediate wake comprises a transport of turbulent energy away from the centreline while the unmixed-fluid contribution implies a transport of turbulent energy towards the centreline (Andreopoulos \& Bradshaw 1980). To the authors' knowledge, the vortical structures providing the means of these opposite transport processes have 
not been yet identified. The intermediate wake is identified by the logarithmic increase of the time-averaged centreline velocity (Haji-Haidari \& Smith 1988) and extends from $25 \theta_{0}$ to $350 \theta_{0}$ according to Ramaprian et al. (1982). The growth rate of the wake width and the decay rate of the centreline defect are slower than both the near wake $\left(x<25 \theta_{0}\right)$ and the following far wake $\left(x>350 \theta_{0}\right)$. The near wake and the intermediate wake form the developing wake region, which is covered in this work.

The asymptotic state or the far wake follows the developing wake region once the mixing process between the two boundary layers is completed. At this downstream location, an asymptotic state is reached and the structural parameters change from values typical of a boundary layer or near wake to values typical of a self-preserving far wake (Andreopoulos \& Bradshaw 1980). The asymptotic state is reached at quite large distances $\left(x>350 \theta_{0}\right)$ from the trailing edge where the growth rates are well predicted by classical fully developed far-wake analysis and simple turbulence models such as constant eddy viscosity (Ramaprian et al. 1982).

The current work aims to tackle the detailed flow physics in the developing wake of the sharp trailing edge of a non-lifting aerofoil. Specifically, the work is conducted to identify the unsteady organization of the coherent flow structures in the wake region along with their evolution from the initial organization of the upstream turbulent boundary layer. The results will shed light on the response of the coherent structures of a turbulent boundary layer as a result of change in the boundary condition and also elucidates the mixing process in the turbulent wake of a sharp trailing edge. The identified coherent structures of the wake region should satisfy the observations of the previous literature.

(i) The profiles of statistical quantities of the wake such as the mean and fluctuating velocity have similar trend with respect to the turbulent boundary layer prior to the trailing edge (Haji-Haidari \& Smith 1988). The length scales available in the turbulent boundary layer and the subsequent wake are also of the same order of magnitude. This suggests that the coherent structures of the wake region should have certain similarities to the structures of the upstream boundary layer.

(ii) The net transport of momentum within the wake region is oriented towards the wake centreline in order to recover the wake deficit (Andreopoulos \& Bradshaw 1980). This is in contrast to a fully developed turbulent boundary layer as it is dominated by ejection events that transport momentum away from the wall vicinity resulting in further growth of the boundary layer thickness. Therefore, the vortical structures that carry out the transport of momentum within the wake region act in an opposite direction to those of the turbulent boundary layer.

(iii) The production of turbulence persists downstream of the trailing edge as there is considerable $u$ and $v$ correlation in this region (Andreopoulos \& Bradshaw 1980). This indicates that the dominant coherent structures in the wake region contribute to $u$ and $v$ correlation and production of Reynolds shear stress.

(iv) The existence of the logarithmic trend along the wake centreline in the intermediate wake region (Haji-Haidari \& Smith 1988) suggests the possibility of the existence of self-similar structures with a hierarchy of length scales in this region.

The difficulties in identifying the spatio-temporal organization of the coherent structures are overcome in this work by applying state-of-the-art time-resolved tomographic particle image velocimetry (Tomo-PIV), which is a three-dimensional extension of standard PIV (Elsinga et al. 2006). The Tomo-PIV technique provides a three-dimensional matrix of the velocity vectors to demonstrate quantitatively the 


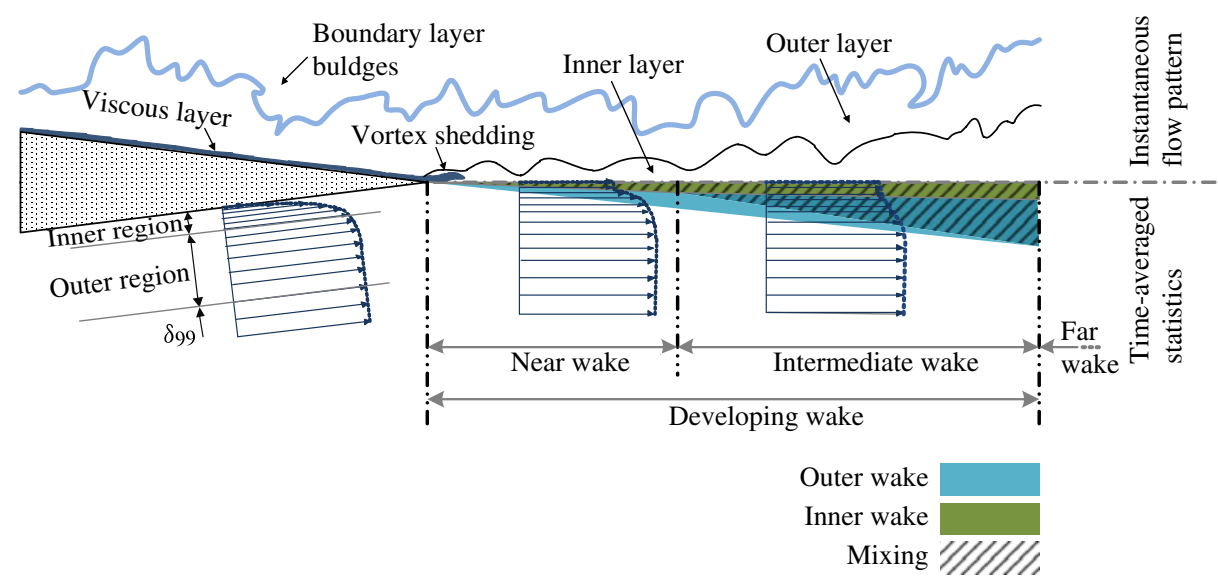

FIGURE 1. (Colour online available at journals.cambridge.org/flm) A schematic of the turbulent flow in the vicinity of a sharp trailing edge at zero angle of attack. The upper half represents the instantaneous flow pattern, while the lower half shows the time-averaged characteristics of the wake regions.

organization of the turbulent structures, and has been previously applied to investigate different flow fields. Schröder et al. (2008) have conducted a study of coherent structures within a turbulent boundary layer using a time-resolved Tomo-PIV system and observed the low-speed streaks and hairpin-like vortical structures. Humble, Elsinga, Scarano \& Van Oudheusden (2009) have proposed a conceptual model of the three-dimensional unsteady flow organization of a shock wave/turbulent boundary layer interaction from Tomo-PIV measurement. This measurement technique has been further applied by Elsinga et al. (2010) to characterize the hairpin vortices of a supersonic turbulent boundary layer. The vortex organization in the wake of a cylinder has also been investigated using the Tomo-PIV technique by Scarano \& Poelma (2009). The three-dimensional velocity information obtained from the Tomo-PIV experiment is used in this work to investigate the turbulent boundary layer and the subsequent wake of a symmetric sharp trailing edge.

The methodology of this investigation, consisting of the experimental facility, the measurement and the analysis techniques, are detailed in $\S 2$. The statistical properties of the turbulent boundary layer and the following wake are scrutinized in $\S 3$, which is followed by a study of the unsteady organization of the coherent structures of these regions in $\S 4$. The temporal evolution of the flow structures over the trailing edge are investigated in $\S 5$. Finally, a discussion on the coherent structures of the wake region and their role in the development of the wake is provided in $\S 6$.

\section{Experimental apparatus and procedures}

\subsection{Flow configuration}

An open test-section open-loop low-speed wind tunnel located at the Aerodynamics Department of Delft University of Technology has been used to perform the experiments. The wind tunnel has a rectangular cross-section of $0.40 \mathrm{~m} \times 0.40 \mathrm{~m}$ following a contraction ratio of $8: 1$. A free stream with a turbulence intensity of $0.1 \%$ is generated in the test section by a centrifugal fan driven by a $16.5 \mathrm{~kW}$ electric d.c. motor (Tummers 1999). 
The experiments were carried out on a NACA0012 aerofoil manufactured in aluminium by automated milling. The original NACA0012 of $C=0.4 \mathrm{~m}$ chord length and $14^{\circ}$ total angle at the trailing edge has been modified by extending the trailing edge $4 \mathrm{~mm}$ in the chordwise direction to reduce the trailing-edge thickness to $h=0.2 \mathrm{~mm}$ in order to mitigate vortex shedding. The aerofoil was installed at zero angle of attack spanning the entire test section. The laminar to turbulent transition of the boundary layers on both sides of the aerofoil were forced with a zig-zag strip, to ensure spanwise uniform boundary layer properties. The zig-zag element is $0.5 \mathrm{~mm}$ thick with a pitch of $6 \mathrm{~mm}$ and was located at $0.25 \mathrm{C}$ from the aerofoil leading edge.

At a free stream velocity of $U_{\infty}=14 \mathrm{~m} \mathrm{~s}^{-1}$, the chord-based Reynolds number is $R e_{c}=386000$ and that based on momentum thickness is approximately $R e_{\theta}=1300$ at the trailing edge. The latter ensures a stable turbulent boundary layer at the aerofoil trailing edge while enabling a time-resolved measurement. Two coordinate systems as shown in figure 2 compatible with the boundary layer and the wake are used in this work. The $s-n$ plane of the boundary layer coordinate system is attached to the aerofoil surface, while the $x-y$ plane follows the wake orientation.

The characteristics of the boundary layer at an upstream location of the trailing edge $(s=-20 \mathrm{~mm})$ and also at the trailing-edge location are presented in table 1 . The data are obtained by two-component particle image velocimetry (2C-PIV) measurement and are processed through an algorithm using the average of correlation maps as detailed in the next section. The boundary layer thickness $\left(\delta_{99}\right)$, the displacement thickness $\left(\delta^{*}\right)$ and the momentum thickness $(\theta)$ increase as the flow approaches the trailing edge. It is verified that the trailing edge can be considered 'sharp' as the $h / \delta^{*}=0.09$ at the trailing edge is smaller than the 0.3 limit suggested by Blake (1986). The momentum thickness $\left(\theta_{0}=1.7 \mathrm{~mm}\right)$ and other parameters at the trailing edge $(s=0)$ are used in this work to obtain non-dimensional coordinates.

The shape factor at the $s=-20 \mathrm{~mm}$ location is $H=1.57$, which is close to the recorded value of $H=1.6$ by Haji-Haidari \& Smith (1988) for a flat plate of $8^{\circ}$ included angle at the trailing edge and $H=1.54$ by Hebbar (1986) for an included angle of $19^{\circ}$. The investigations using models with smaller thickness and included angle at the trailing edge have recorded lower values of shape factor. Ramaprian et al. (1982) have measured $H=1.29$ for a flat plate of $1.8^{\circ}$ trailing-edge included angle and Andreopoulos \& Bradshaw (1980) measured $H=1.38$ for a flat plate of $3.5^{\circ}$ trailing-edge included angle. The difference is due to the higher pressure gradient caused by the trailing-edge taper.

An estimation of the smallest and largest scales of the coherent structures within the turbulent boundary layer is necessary to design and evaluate the measurement system. The smallest coherent structures that have a lifetime long enough to contribute to statistics have a diameter of approximately 20 times the wall units $\left(20 \lambda^{+}\right)$, which is approximately $600 \mu \mathrm{m}$ at $s=-20 \mathrm{~mm}$ location (Stanislas, Perret \& Foucaut 2008). The largest structures in a turbulent boundary layer that are considered to be resolved here are the hairpin packets or the large-scale motions (LSMs) with typical maximum streamwise extent of $3 \delta$ (Adrian et al. 2000; Guala, Hommema \& Adrian 2006), equivalent to approximately $30 \mathrm{~mm}$ in the present experiment. Therefore, in the current experiment the size ratio of the LSMs to the smallest coherent structures is $W_{s t r}=50$, which should be satisfied by the dynamic range of the measurement system (Herpin et al. 2008). 


\begin{tabular}{lccc}
\multicolumn{2}{l}{ Parameter } & At $s=-20 \mathrm{~mm}$ & At $s=0$ \\
$U_{\infty}$ & $\left(\mathrm{m} \mathrm{s}^{-1}\right)$ & 14 & 14 \\
$\delta_{99}$ & $(\mathrm{~mm})$ & 10.1 & 11.9 \\
$\delta^{*}$ & $(\mathrm{~mm})$ & 2.2 & 3.0 \\
$\theta$ & $(\mathrm{mm})$ & 1.4 & 1.7 \\
$\operatorname{Re}_{c}$ & - & 386000 & 386000 \\
$\operatorname{Re}_{\delta 99}$ & - & 9400 & 11000 \\
$\operatorname{Re}_{\theta}$ & - & 1300 & 1600 \\
$u_{\tau}$ & $\left(\mathrm{m} \mathrm{s}^{-1}\right)$ & 0.49 & 0.41 \\
$\lambda^{+}$ & $(\mu \mathrm{m})$ & 30 & 36 \\
$\delta^{+}$ & - & 338 & 331 \\
$H$ & - & 1.57 & 1.76
\end{tabular}

TABLE 1. Boundary layer parameters at an upstream location of the trailing edge $(s=-20 \mathrm{~mm})$ and at the trailing edge $(s=0)$.

\subsection{Two-component particle image velocimetry}

A 2C-PIV system has been applied to investigate the turbulent statistics and the twodimensional structure of the flow field. This measurement system has been repeatedly used for characterization of vortical structures in different experiments investigating turbulent boundary layers (e.g. Adrian et al. 2000; Stanislas et al. 2008).

The required illumination for the PIV experiment is provided by a Quantronix Darwin-Duo laser system with average output of $80 \mathrm{~W}$ and $2 \mathrm{~mJ} \times 13 \mathrm{~mJ}$ per pulse energy at $3 \mathrm{kHz}$ operation frequency (527 nm wavelength). The laser beam diameter is $3 \mathrm{~mm}$ at the output and has a pulse width of approximately $150 \mathrm{~ns}$. A laser sheet of approximately $1 \mathrm{~mm}$ thickness was formed by a combination of two spherical lenses of $f=+200$ and $-150 \mathrm{~mm}$ focal length and a cylindrical lens of $f=-80 \mathrm{~mm}$. A Photron Fast CAM SA1 with a 12-bit complementary metal-oxide-semiconductor sensor of $1024 \times 1024$ pixels (pixel pitch $20 \mu \mathrm{m}$ ) was used to image the illuminated region. The camera was equipped with a Nikon objective of $f=105 \mathrm{~mm}$ focal length set to an aperture of $f / 2.8$. The plane of focus was slightly offset with respect to the illumination plane (defocusing) to obtain a blurred spot spanning approximately two pixels, which mitigates bias errors associated with pixel locking (Westerweel 1997). Global seeding of the test section was conducted with $1 \mu \mathrm{m}$ droplets generated using a SAFEX smoke generator placed in the plenum of the wind tunnel.

The 2C-PIV measurements were conducted over a wall-normal streamwise field of view (FOV) of $52.3 \mathrm{~mm} \times 52.3 \mathrm{~mm}$. Ensembles of 1000 double-frame recordings with $42 \mu \mathrm{s}$ pulse separation at acquisition frequency of $100 \mathrm{~Hz}$ have been acquired for statistical investigation corresponding to an integration time of $10 \mathrm{~s}$. The images have been processed using the LaVision software Davis 7.4 with two different methods for the mean velocity profiles and the turbulent fluctuations. The velocity profiles are obtained by averaging the cross-correlation maps over the available ensemble of recordings in order to enhance the convergence of the statistics (Meinhart, Wereley \& Santiago 2000). This method increases the signal-to-noise ratio especially in the high-velocity-gradient region close to the wall, making it feasible to use interrogation windows of $6 \times 6$ pixels with $75 \%$ overlap for statistical evaluation. The system parameters are shown in table 2 . The spatial dynamic range (SDR) of this configuration in the streamwise direction is 85 if the smallest resolvable spatial variation is considered to be twice the size of the interrogation window. The high 

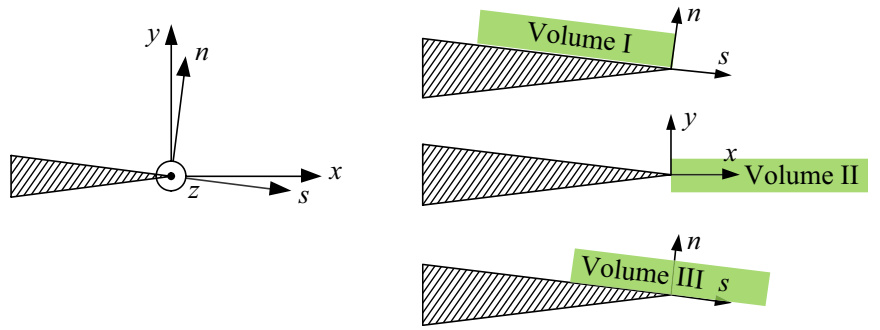

FIGURE 2. (Colour online) The surface-aligned $(n-s)$ and the wake-aligned $(x-y)$ coordinate systems and the two-dimensional projection of the Tomo-PIV measurement domains. The spanwise extension of the measurement domain along $z$ is $47 \mathrm{~mm}$.

SDR of this configuration and the magnification of $M=0.39$ will cover the smallest and the largest desirable structures within the boundary layer but only statistics are available due to the processing scheme. On the other hand, the turbulent fluctuations are obtained from individual correlation maps through processing the images by windows of $16 \times 16$ pixels and $50 \%$ overlap. This configuration offers an SDR of 32 resulting from an interrogation window size of $27.2^{+}(0.81 \mathrm{~mm})$ and a FOV of $5.2 \delta_{99}$ $(52.3 \mathrm{~mm})$ in the streamwise direction. The spatial resolution of this PIV configuration in relation to the considered flow field is relatively high, as $\mathrm{SDR} / W_{s t r}=0.64$ is equal to that of Herpin et al. (2008) achieved by a stereoscopic PIV setup of high spatial dynamic range.

\subsection{Tomographic particle image velocimetry}

The Tomo-PIV system provides quantitative flow visualization to assess the threedimensional characteristics of the coherent structures in the flow field. This measurement method is a three-dimensional extension of planar (stereoscopic) PIV and was developed by Elsinga et al. (2006). The illuminated volume in a Tomo-PIV experiment is simultaneously imaged from several viewing directions (typically three to six). A target calibration is initially conducted to establish the first estimation of the relation between the image coordinates and the physical space. The calibration errors are further reduced to a fraction of a pixel using the self-calibration method (Wieneke 2008). The images are then processed by the multiplicative algebraic reconstruction technique (MART) algorithm (Herman \& Lent 1976) to reconstruct the three-dimensional light intensity distribution. Finally, a three-dimensional crosscorrelation with multigrid, iterative volume deformation (VODIM; Scarano \& Poelma 2009) is applied to the reconstructed particle distribution of two subsequent exposures to obtain the instantaneous velocity field.

The Tomo-PIV measurement system consists of the same base equipment (laser, cameras, lens objective, particle generator) as the 2C-PIV system. The higher light demand of the Tomo-PIV experiment was fulfilled using a multi-pass light amplification system. The system illuminated a measurement volume of $8 \mathrm{~mm}$ thickness ( $y$ direction) and $50 \mathrm{~mm}$ width ( $x$ or $s$ direction) by successive reflections of the initial laser beam in between two mirrors. A schematic drawing of the illumination systems is shown in figure 3. This multi-pass light amplification was first applied in Tomo-PIV experiments by Schröder et al. (2008) and a detailed description is given by Ghaemi \& Scarano (2010).

The imaging system consists of four Photron Fast CAM cameras aligned along the wall-normal spanwise plane with respect to the aerofoil, as illustrated in figure 3 . 
Cameras 1 and 4 are inclined at $40^{\circ}$, and cameras 2 and 3 have $15^{\circ}$ angle with respect to the wall-normal direction. The cameras are equipped with Scheimpflug adapters and $105 \mathrm{~mm}$ objectives. The aperture of the two side cameras (1 and 4) is set to $f / 22$ while the two middle cameras ( 2 and 3 ) image at $f / 16$. This aperture setting compensates for the differences in scattered light towards the four cameras, yet bounding the illuminated volume within the depth of focus of each camera. The four cameras and the laser have been synchronized to record double-frame images with a $42 \mu$ s laser pulse separation at 100 and $2700 \mathrm{~Hz}$ using a high speed controller (LaVision). The illumination volume has been seeded with the $1 \mu \mathrm{m}$ droplets to obtain a concentration of 4 particles $\mathrm{mm}^{-3}$, resulting in a source density of approximately 0.065 particles per pixel in the images corresponding to the measurement volume of $47 \mathrm{~mm} \times 47 \mathrm{~mm} \times 8 \mathrm{~mm}$.

The data analysis associated with the Tomo-PIV technique has been conducted by Davis 7.4 on an eight-core personal computer. The images were preprocessed by subtracting the minimum value of each pixel along the sequence. Subsequently, the residual background intensity is removed by subtracting the sliding minimum with kernel of 31 pixels. The image intensity was normalized by the average over a kernel of 51 pixels. The reconstructed volume dimensions in streamwise $(x)$, spanwise $(z)$ and wall-normal $(y)$ coordinates were $47 \mathrm{~mm} \times 47 \mathrm{~mm} \times 8 \mathrm{~mm}$, which was discretized at 22 voxels $\mathrm{mm}^{-1}$. Interrogation volumes of $32 \times 32 \times 32$ voxels with $75 \%$ overlap have been considered, enclosing approximately 13 particles. This results in SDR $=16$ and $\mathrm{SDR} / W_{s t r}=0.32$ from an interrogation window size of $48.9^{+}(1.47 \mathrm{~mm})$ and a FOV extent of $4.7 \delta_{99}(47 \mathrm{~mm})$ in the streamwise direction. The vector field is calculated by cross-correlation adopting a multi-grid volume deformation method. The obtained vector field is a volume of $128 \times 128 \times 22$ vectors each spaced $0.37 \mathrm{~mm}$ (corresponding to 12 wall units) away from the neighbouring vectors.

\subsection{Pattern recognition analysis}

The detection of relevant features exhibiting certain characteristics is performed by means of pattern recognition analysis (PRA). The approach follows that used by Ferré \& Giralt (1989) and later modified for PIV measurements by Scarano, Benocci \& Riethmuller (1999). The working principle relies on the choice of an indicator function and on the formulation of a spatial template. For this purpose, a priori knowledge about the flow properties is required from a preliminary evaluation of the measurements. The inspection returns hairpin-like vortices in the turbulent boundary layer before the trailing edge as shown in the remainder. The hairpins are best visualized by making use of the $Q$-criterion (Hunt, Wray \& Moin 1988) based on the second eigenvalue of the velocity gradient tensor. Moreover, the hairpins are associated with a strong ejection events (Q2 in the quadrant splitting scheme introduced by Wallace et al. (1972)). Therefore, here a second indicator function is also applied as $u v<0$ while $u<0$ and $v>0$ to detect the ejection events. In this work, the PRA technique is merely applied to obtain an ensemble average of the detected structures. The technique is not aimed at proving the existence of a specific group of turbulent structures since the result can be biased to the choice of the indicator function.

The spatial template follows an arc-like vortex pattern inclined with respect to the wall-normal direction. The main parameters governing the topology are the width $g$ between the legs and the height $h$ indicated in figure 4 . The hairpin width is reported by Head \& Bandyopadhyay (1981) to lie in the range of $g=10-100^{+}$depending on the wall-normal location and the Reynolds number. In the present case a value of $120^{+}(3.6 \mathrm{~mm})$ is chosen in accordance with the Tomo-PIV visualizations, which is 


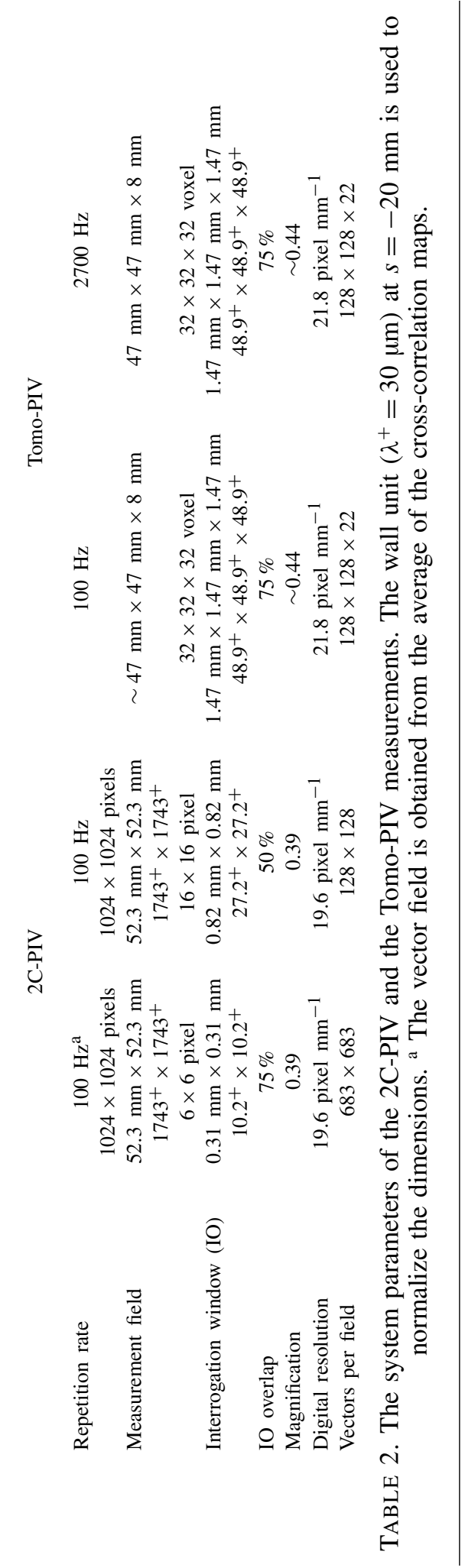




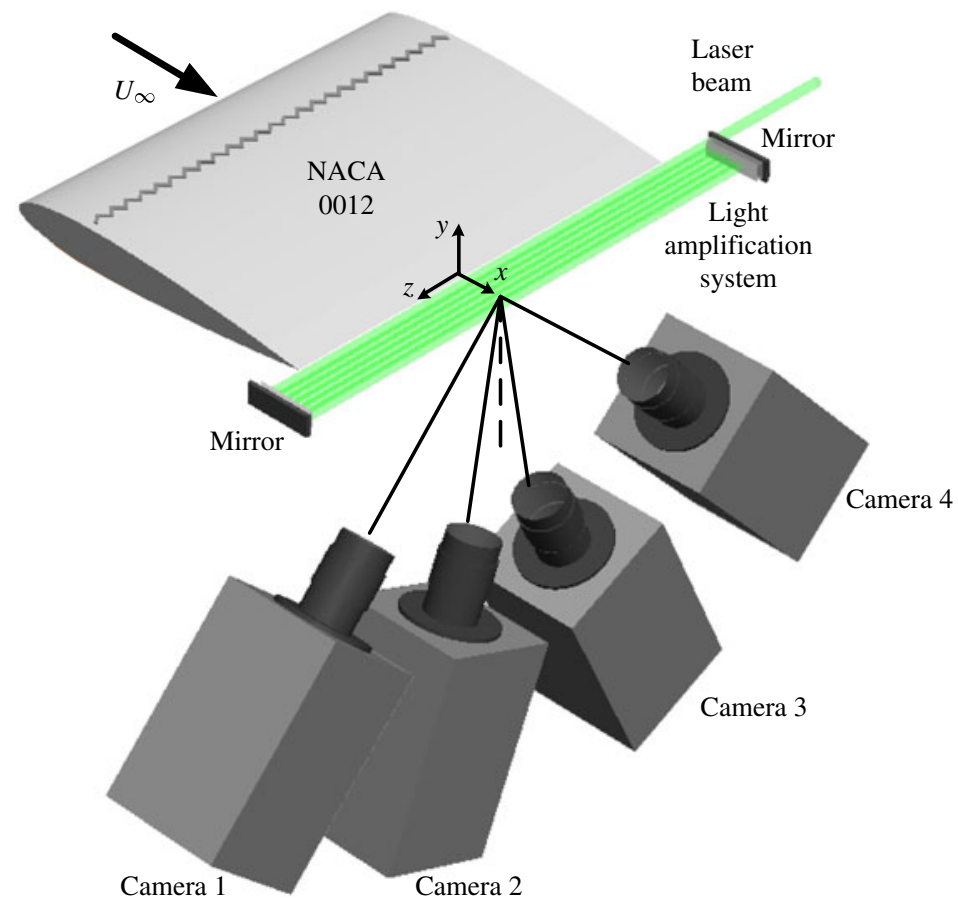

FIgURE 3. (Colour online) The arrangement of the cameras, aerofoil and the multi-path illumination system in the Tomo-PIV experiment.

also consistent with the typical width of the low-speed streaks. From the observations, it emerges that the vortex axis in the head region has a significant flatness, which is evaluated with a fourth-order polynomial for the vortex line. In the case of the coherent structure within the boundary layer, the algorithm applies a reference hairpin shape and the embedded ejection event as the detection criterion. The curve describing the vortex line topology is given by

$$
\left\{\begin{array}{l}
x=\frac{1}{a} z^{4} \cos \alpha \\
y=\frac{1}{a} z^{4} \sin \alpha
\end{array}\right.
$$

where the constant $a$ depends on the boundary layer scale and in this case is set to $a=5 \mathrm{~mm}^{3}$ (equivalent to $\left(167^{+}\right)^{3}$ ). The angle $\alpha$ is the orientation of the hairpin structure with respect to the streamwise direction and is selected as $\alpha=60^{\circ}$. As the boundary layer prior to the trailing edge is subject to an adverse pressure gradient, the choice of the inclination angle is based on visual inspection of sample hairpin vortices and is higher than the $45^{\circ}$ reported for fully developed boundary layers under zero pressure gradient (Head \& Bandyopadhyay 1981). The embedded ejection event is generated by applying $-u v / U_{\infty}=0.01$ (satisfying $u<0$ and $v>0$ ) within an ellipsoid defined by

$$
\frac{x^{2}}{a_{x}^{2}}+\frac{y^{2}}{a_{y}^{2}}+\frac{z^{2}}{a_{z}^{2}}=1
$$


The constants are set as $\alpha_{x}=1.5 \mathrm{~mm}\left(50^{+}\right), \alpha_{y}=3.5 \mathrm{~mm}\left(117^{+}\right)$and $\alpha_{z}=$ $3.5 \mathrm{~mm}\left(117^{+}\right)$. The spatial pattern of the template is shown in figure 4(a) by means of iso-surfaces of $Q=1 \times 10^{6} \mathrm{~s}^{-2}$ and $-u v / U_{\infty}=0.008$. The indicator function for the wake region is considered as the combination of a counter-hairpin vortex that has a reverse topology with respect to the hairpin vortex and encloses a sweep event $-u v / U_{\infty}=0.008$ (satisfying $u>0$ and $v<0$ ). This indicator is anticipated by visual inspection of the Tomo-PIV snapshot, which is followed by verification through the algorithm. The selection of the counter-hairpin vortex will be discussed in detail in $\S 4.2$.

The three-dimensional spatial cross-correlation function is evaluated between the chosen detector and the scalar field of $Q$. The operation is similarly made for the distribution of $u v$. A single detection criterion $C_{H}$ is obtained by multiplication of the two correlation coefficients, $C_{Q}$ and $C_{v}$. The detection condition for structures of significant strength is $C_{H}>0.1$, with a minimum width of the correlation peak of five grid nodes.

The conditional averaged structural properties of the hairpin and counter-hairpin events are based on the ensemble of the detected events after aligning them to the closest grid node. The ensemble-averaged structure may also be taken as a new reference template for further iterations in the detection algorithm (Ferré \& Giralt 1989). In the present study, however, further iterations do not appear to bring significant differences in the structure of the ensemble-averaged hairpin.

\section{Statistical analysis}

\subsection{Boundary layer and wake recovery}

The slight adverse pressure gradient prior to the trailing edge of NACA0012 results in an increase of the boundary layer thickness as the flow approaches the trailing edge, as observed in the velocity profile of figure $5(a)$. The profile at $s / \theta_{0}=-12$ is shown for both the upper and lower surfaces, indicating the symmetry of the mean flow. The log layer is observed to have a higher slope in comparison to a fully developed boundary layer in the semi-logarithmic plot of figure 5(b). This difference has also been observed closely upstream of the trailing edge in the boundary layer profiles of Haji-Haidari \& Smith (1988) and Sagrado, Hynes \& Hodson (2006) and ascribed to the non-equilibrium of the boundary layer in the vicinity of the trailing edge.

The recovery of the average velocity profile across the wake region is shown in figure $6(a)$. The large deficit of the inner wake observed at $x / \theta_{0}=0.3$ is filled to a large extent within the first few $\theta_{0}$ of the wake development, while the change in the outer layer is negligible, indicating that the mixing process is confined to the inner layer. The delayed response of the outer layer to the recovery of the inner layer deficit is also observed in the velocity profiles of Hamelin \& Alving (1996). The centreline velocity at $x / \theta_{0}=3.5$ is $0.4 U_{\infty}$ while the outer layer is almost unchanged, exhibiting the characteristics of the near wake region. At $x / \theta_{0}>5$ the velocity profile at the outer layer of the wake also starts to increase, which is due to the curvature of the streamlines in the vicinity of the trailing edge. At $x / \theta_{0}=12$ and 24 both the inner layer and the outer layer profiles have developed towards the equilibrium condition of the far field. The lower half of the wake profile at $x / \theta_{0}=12$ reveals the wake symmetry.

The boundaries of different wake regions can be more accurately identified from a semi-logarithmic plot of the wake centreline velocity as shown in figure $6(b)$. The inner scaling of this plot is based on the viscous length scale along the wake 


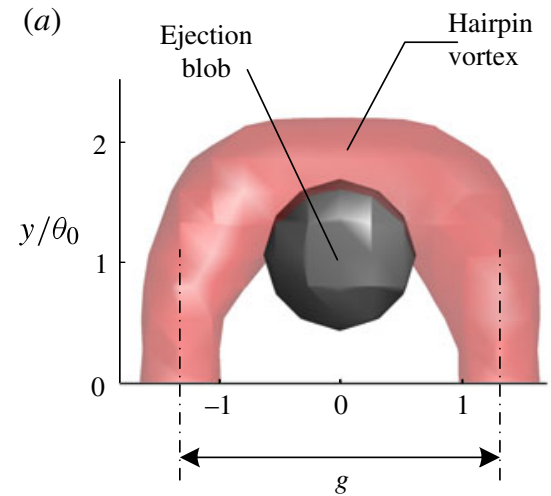

(b)

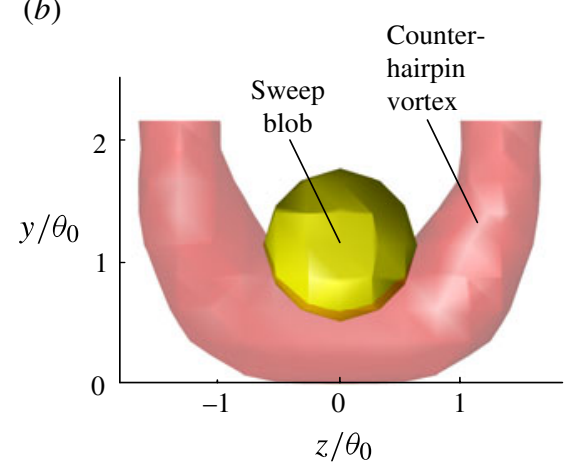

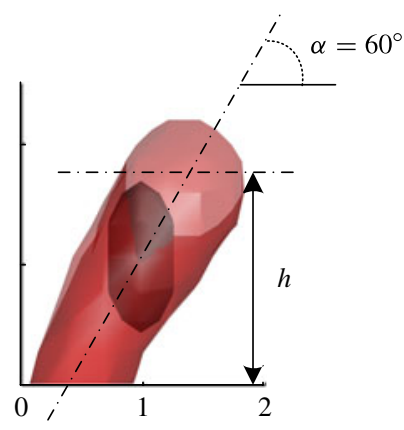

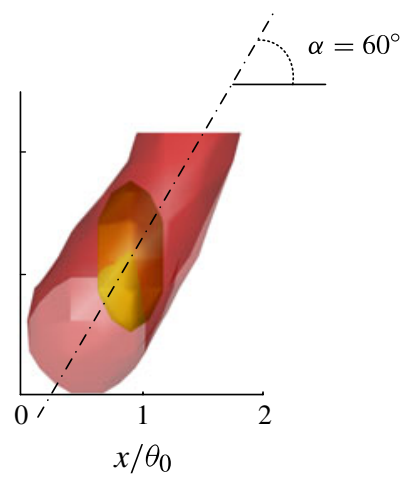

FIgURE 4. (Colour online) (a) The reference structure for hairpin detection within the boundary layer and $(b)$ the reference structure for the wake. The (U-shaped) red (mid-grey) iso-surface shows the vortex surface at $Q=1 \times 10^{6} \mathrm{~s}^{-2}$, while dark grey and yellow (light grey) iso-surfaces, respectively, indicate an ejection event $(u<0$ and $v>0)$ and a sweep event $(u>0$ and $v<0)$, both of $-u v / U_{\infty}^{2}=0.008$ strength.

centreline, which is equal to $\lambda^{+}=93 \mu \mathrm{m}$. The centreline velocity exhibits a linear behaviour immediately after the trailing edge till $x^{+}<15$, similar to the viscous sublayer of a turbulent boundary layer. The linear region is followed by a buffer interval where a transition towards logarithmic behaviour is observed. The latter begins at $x^{+}=50$ and extends through the rest of the measurement domain. Different boundaries have been suggested for the wake regions in previous literature (Ramaprian et al. 1982; Hebbar 1986; Haji-Haidari \& Smith 1988). The discrepancy is due to the fact that the scaled centreline velocity depends on the Reynolds number, as the large eddies of the outer layer influence the small-scale mixing within the inner layer (Nakayama \& Liu 1990).

The qualitative similarity of the semi-logarithmic plot across the boundary layer and along the wake centreline requires further attention. The initial linear relation is due to the viscosity-dominated flow in both the near-wall region (viscous sublayer) and also in the immediate wake of the trailing edge. The instantaneous velocity fields of the near wake show two viscous sublayers of the lower and upper surface boundary layers merging at the trailing edge and forming a slightly oscillating laminar wake, as illustrated in figure 1. In a turbulent boundary layer, the logarithmic portion represents a time-averaged contribution of self-similar structures retaining a linear variation of length scale (Adrian et al. 2000); mostly hairpin vortices form a hierarchy of different 

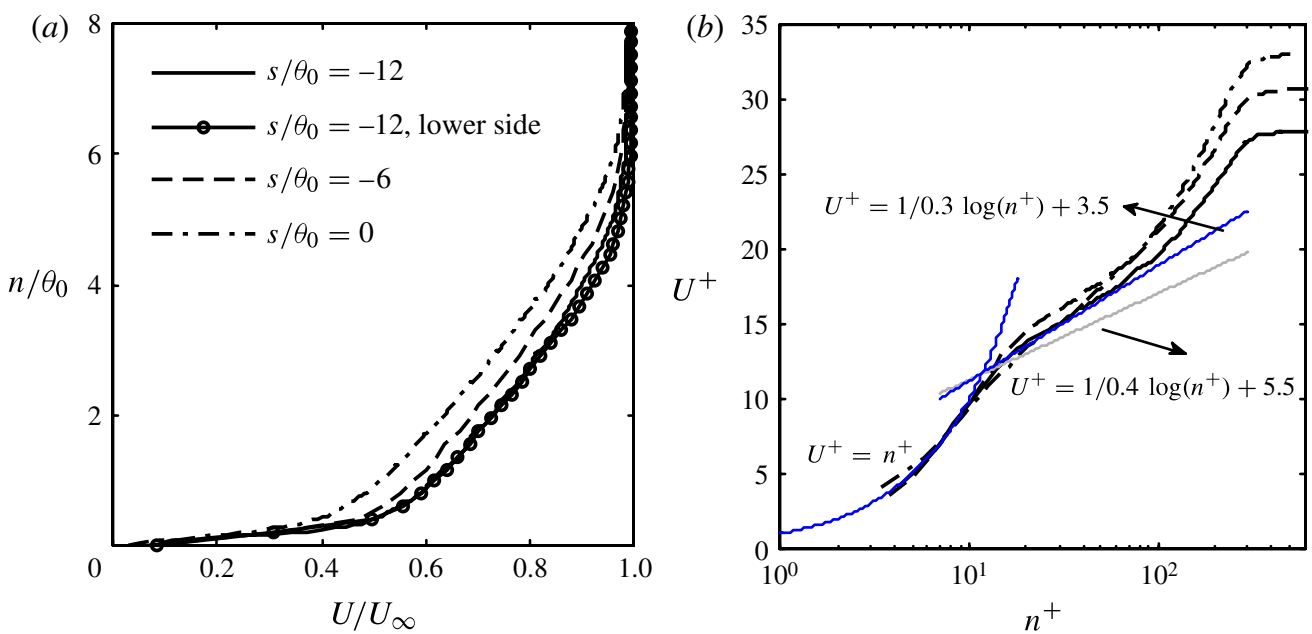

FIGURE 5. (Colour online) The average velocity profile in the boundary layer prior to the trailing edge in $(a)$ linear and $(b)$ semi-log scale. The linear fit to the sublayer and the logarithmic fit to the log layer is also shown. The lower logarithmic fit corresponds to a fully developed turbulent boundary layer at zero pressure gradient (White 1974).
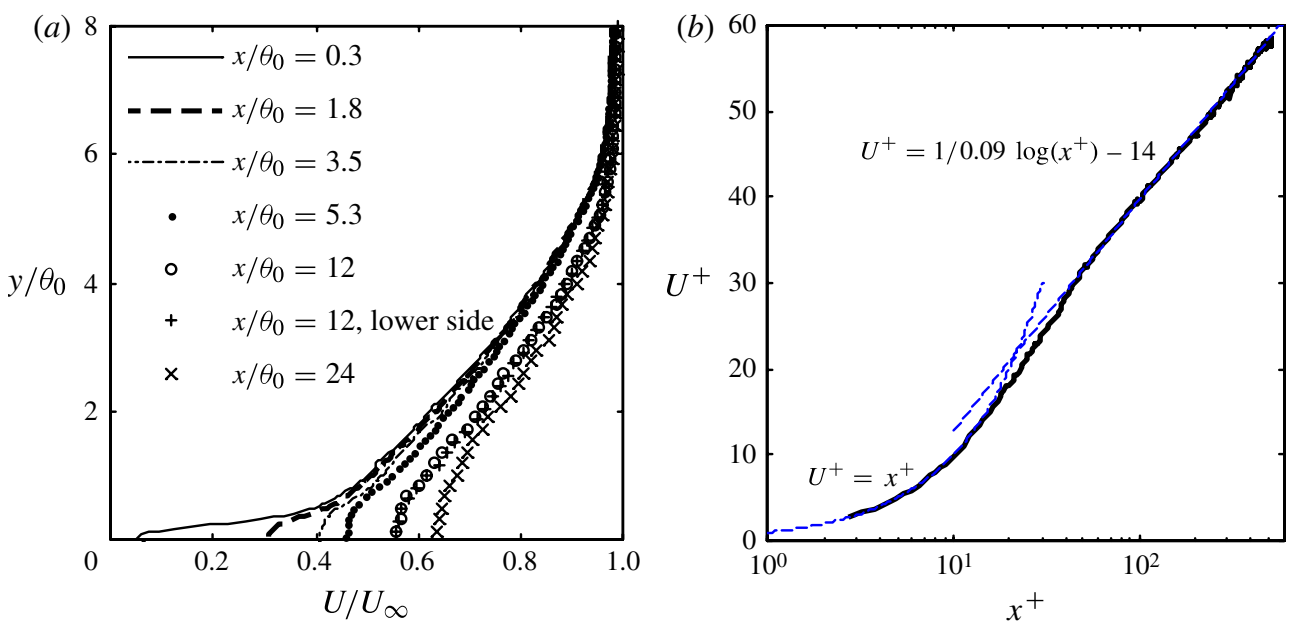

FIGURE 6. (Colour online) (a) Streamwise velocity profiles across the wake at several streamwise locations. (b) Mean streamwise velocity along the wake centreline in semi-log scale. The dashed lines are curve fits to the linear and logarithmic layers.

sizes as a result of the stretching imposed by the mean shear across the boundary layer (Adrian 2007). The existence of the logarithmic trend along the wake centreline also indicates the possibility of self-similar behaviour in the intermediate wake region, as will be discussed in $\S 6$.

\subsection{Production and transport of turbulence}

The normal and shear components of Reynolds stress are reported here in the interval encompassing the turbulent boundary layer and the intermediate wake region. Investigation of the normal components, $\left\langle u^{2}\right\rangle$ and $\left\langle v^{2}\right\rangle$, can express the variation in 

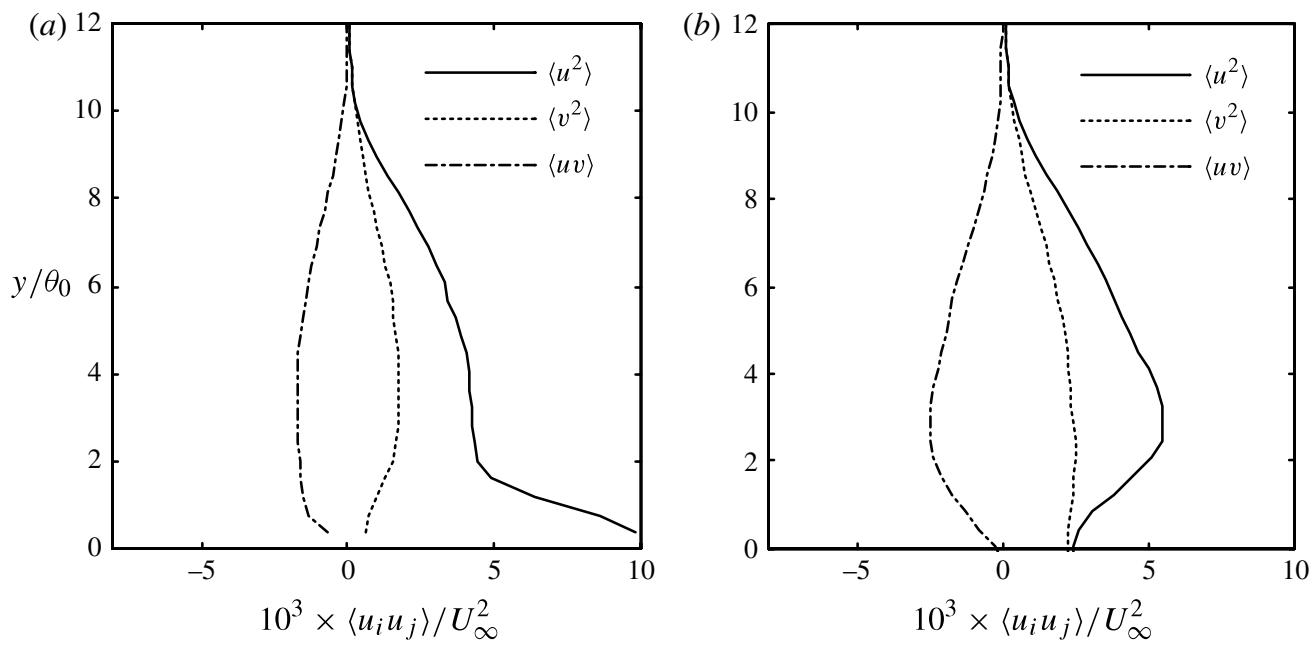

FIGURE 7. Normal and shear Reynolds stress $(a)$ in the turbulent boundary layer at $s / \theta_{0}=-12$ and $(b)$ along the centreline of the following wake at $x / \theta_{0}=12$.

turbulence kinetic energy across the trailing edge, while the Reynolds shear stress $\langle u v\rangle$ depicts the flux of momentum transported by turbulent fluctuations and is also relevant to the acoustic analysis of the trailing-edge noise (Ffowcs Williams \& Hall 1970).

The trends of the profiles of Reynolds stress prior to the trailing edge at $s / \theta_{0}=-12$ shown in figure $7(a)$ are similar to a fully developed turbulent boundary layer (Spalart 1988). The restraining boundary condition of the solid surface results in lower wall-normal fluctuations, $\left\langle v^{2}\right\rangle$, in comparison to streamwise fluctuations, $\left\langle u^{2}\right\rangle$. The maximum in all the profiles corresponds to a turbulence intensity peak at the buffer layer. The shear stress $\langle u v\rangle$ is mainly associated with ejection events induced by hairpin vortices (Wallace et al. 1972). The behaviours of $\left\langle u^{2}\right\rangle,\left\langle v^{2}\right\rangle$ and $\langle u v\rangle$ at $x / \theta_{0}=12$ of the wake region in figure $7(b)$ resembles those of the upstream turbulent boundary layer with approximately the same intensity. The maximum of $\langle u v\rangle$ is at $y / \theta_{0} \approx 3$; consequently, the net force exerted by the Reynolds shear stress, $\mathrm{d}(-\rho\langle u v\rangle) / \mathrm{d} y$, is negative and uniform above this point and positive below it. While the negative force of the outer layer decelerates the mean velocity, the positive force of the inner wake accelerates the flow and acts to fill the velocity deficit at the wake centreline. The disappearance of the wall results in rapid intensification of the $\left\langle v^{2}\right\rangle$ fluctuations (close to the centreline) in comparison to the upstream turbulent boundary layer. The near-wall peak in the $\left\langle u^{2}\right\rangle$ profile of the boundary layer has also vanished in the wake since the high gradient of the near-wall velocity profile has been recovered within a few $\theta_{0}$ from the trailing edge (see figure $6 a$ ). A similar behaviour is observed in the measurements of Hamelin \& Alving (1996) after removing the mean shear from the wall vicinity. At $x / \theta_{0}=12$ and on the centreline, $\left\langle u^{2}\right\rangle=1.1\left\langle v^{2}\right\rangle$, showing development towards an isotropic state of the far wake. There is a considerable level of both $\left\langle u^{2}\right\rangle$ and $\left\langle v^{2}\right\rangle$ fluctuations but $\langle u v\rangle$ is close to zero along the centreline. This suggests that events involving positive and negative $u v$ fluctuations coexist along the wake centreline.

The direction of transport of turbulent kinetic energy by velocity fluctuations before and after the trailing edge is investigated using the triple products as shown in figure $8(a, b)$, respectively. The negative values of $\left\langle u^{3}\right\rangle$ and positive $\left\langle v u^{2}\right\rangle$ 

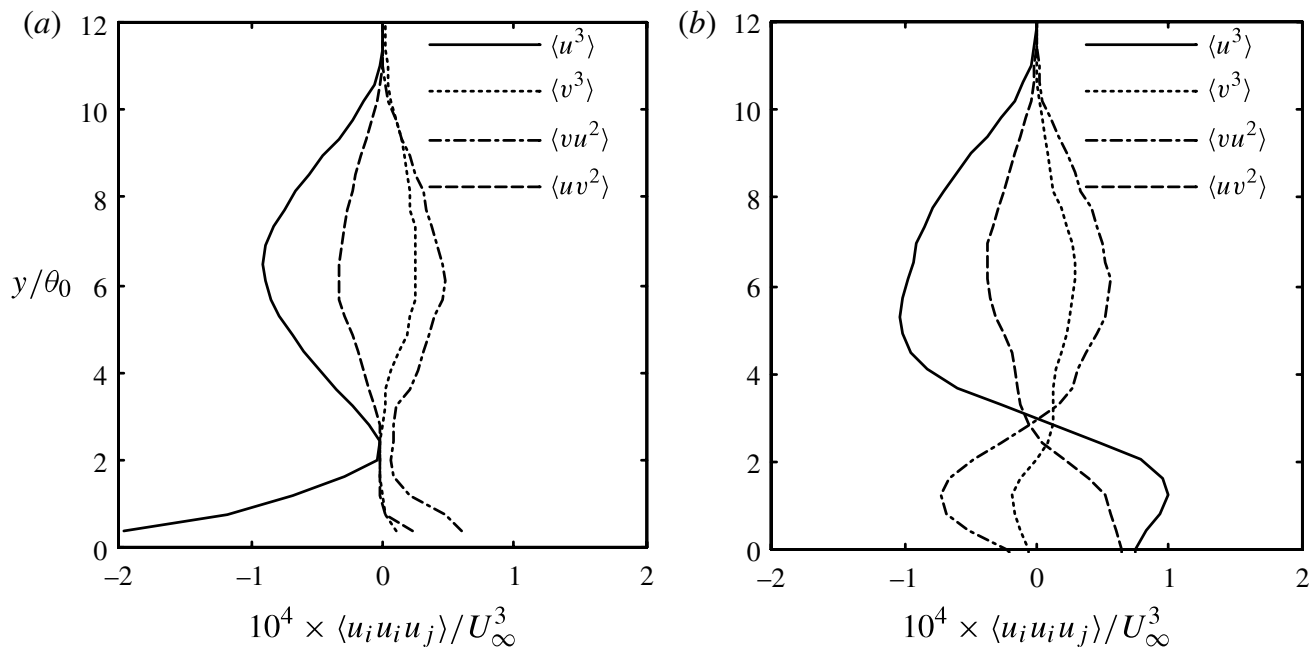

FIGURE 8. Triple products of turbulent fluctuations $(a)$ in the turbulent boundary layer at $s / \theta_{0}=-12$ and $(b)$ along the centreline of the subsequent wake at $x / \theta_{0}=12$.

at $s / \theta_{0}=-12$ indicate that the turbulent kinetic energy embedded in $\left\langle u^{2}\right\rangle$ is transported dominantly via negative $u$ and positive $v$ fluctuations. The $\left\langle u v^{2}\right\rangle$ and $\left\langle v^{3}\right\rangle$ products are also negative and positive, respectively, which also indicate dominant transport of $\left\langle v^{2}\right\rangle$ turbulent kinetic energy through negative $u$ and positive $v$ fluctuations. Although the ensemble of the triple products does not verify that $u<0$ and $v>0$ occur simultaneously, it is known that they are associated with ejection events within the turbulent boundary layer (Corino \& Brodkey 1969). The triple products of turbulent fluctuations in the developing wake location at $x / \theta_{0}=12$ in figure $8(b)$ show a similar behaviour, i.e. $u<0$ and $v>0$, only within the outer wake region $\left(y / \theta_{0}>3\right)$. Therefore, the ejection events of the turbulent boundary layer further persist into the outer layer of the wake and dominate the transport of turbulent kinetic energy. Instead, in the inner wake $\left(y / \theta_{0}>3\right)$ an opposite trend is observed. Positive $\left\langle u^{3}\right\rangle$ and negative $\left\langle v u^{2}\right\rangle$ indicate that the turbulent kinetic energy embedded in $\left\langle u^{2}\right\rangle$ is transported dominantly via positive $u$ and negative $v$ fluctuations. The $\left\langle u v^{2}\right\rangle$ and $\left\langle v^{3}\right\rangle$ products are also positive and negative, respectively, which also point out major transport of turbulent kinetic energy of $\left\langle v^{2}\right\rangle$ through positive $u$ and negative $v$ fluctuations. A similar trend has previously been observed in the temperature-conditioned sampling of Andreopoulos \& Bradshaw (1980). If these $u$ and $v$ fluctuations are correlated, they form a sweep event, which was also observed as inrushes of flow towards the wake centreline by the hydrogen bubble visualization of Haji-Haidari \& Smith (1988). Andreopoulos \& Bradshaw (1980) have demonstrated using temperature-conditioned sampling that the sweep events transport unmixed fluid of the incoming turbulent boundary layers towards the centreline.

In a turbulent boundary layer, a burst sequence of strong ejections transports mass away from the wall, resulting in further growth of the boundary layer thickness (Tardu 1995). This outward mass transfer is partially compensated by sweep motions of longer duration towards the wall. The result of these two events is a correlation of $u$ and $v$ and consequently strong production of turbulence, which further dissipates in turbulent bulges (Carlier \& Stanislas 2005). Contrary to the turbulent boundary layer where the ejections dominate, the triple products demonstrated that within the 
inner wake region the sweep events have a major contribution in the transport of turbulent kinetic energy. A detailed quadrant analysis is proposed to further explore the statistical occurrence of $u$ and $v$ fluctuations within the wake region in comparison to the turbulent boundary layer and verify their correlation.

\subsection{Ejection and sweep events}

The quadrant splitting identifies the contribution of the ejection and sweep events to the total mean values of kinetic energy and Reynolds stress. The $u$ and $v$ within the boundary layer demonstrate coexistence of ejection (Q2 quadrant) and sweep (Q4 quadrant) events at different heights within the log layer of the boundary layer, as shown in figure $9(a-c)$. The contribution of the sweep events to turbulence production decreases on moving away from the wall and the ejections become dominant at the interface of the bulges. Along the wake centreline the major correlation between the $u$ and $v$ fluctuations is observed as sweep events occupying both Q4 and Q1 quadrants of figure $9(d-f)$ since they are generated by both sides of the wake centreline. The Q4 sweep events transport the unmixed flow (Andreopoulos \& Bradshaw 1980) of the upper turbulent boundary layer $(y>0)$ downwards to the wake centreline, while the Q1 transport the unmixed flow of the lower boundary layer $(y<0)$ up towards the centreline. The ejection events along the centreline are fewer in number and weaker in strength. Therefore, the sweep events dominate and transport the unmixed fluid into the inner layer, enhancing the recovery of the wake deficit. The quadrant splitting in the outer layer of the wake is similar to the upstream turbulent boundary layer, as the outer layer mostly consists of unmixed fluid of the boundary layer advected into the wake. This is manifested with the increase in magnitude and frequency of the ejection events at larger distances from the wake centreline in figure $9(g-i)$. Yet, the quadrant analysis does not reveal the properties of vortical structures responsible for the sweeps and ejections. Questions about the specific eddy motion (vortex structure) behind the sweep events require quantitative three-dimensional visualization of the flow field, which will be addressed in $\S 4$.

The effect of suddenly removing the no-slip condition after the trailing edge can be compared to the experiment of Hamelin \& Alving (1996), who investigated the development of the turbulent boundary layer after sudden removal of the inner layer using a moving-wall apparatus. In both cases the term $\left\langle u^{2}\right\rangle$ rapidly decreases from the wall vicinity, which is due to the recovery of the inner layer deficit. However, the permeable boundary condition along the wake centreline results in an increase of $\left\langle v^{2}\right\rangle$ for the present experiment, whereas in the case of the moving wall only a small level of wall-normal fluctuation is maintained.

A rapid recovery of the wake velocity deficit has been observed in the developing wake region along with a high level of turbulent kinetic energy and Reynolds shear stress, indicating persistence of vortical activities into the wake region. The outer wake layer exhibits turbulent properties similar to the upstream turbulent boundary layer with dominant transport of turbulent kinetic energy towards the free stream via ejection events. As will be demonstrated in the next section, this behaviour is caused by the hairpin vortices, which also take part in the production of turbulence. The inner wake region may embed different vortical activities, as it exhibits an opposite transport of turbulent kinetic energy towards the wake centreline involving sweep events. These vortical structures are expected to follow self-similar behaviour and should grow with increasing distance from the trailing edge as suggested by the logarithmic trend of centreline velocity. The characterization of the topological 

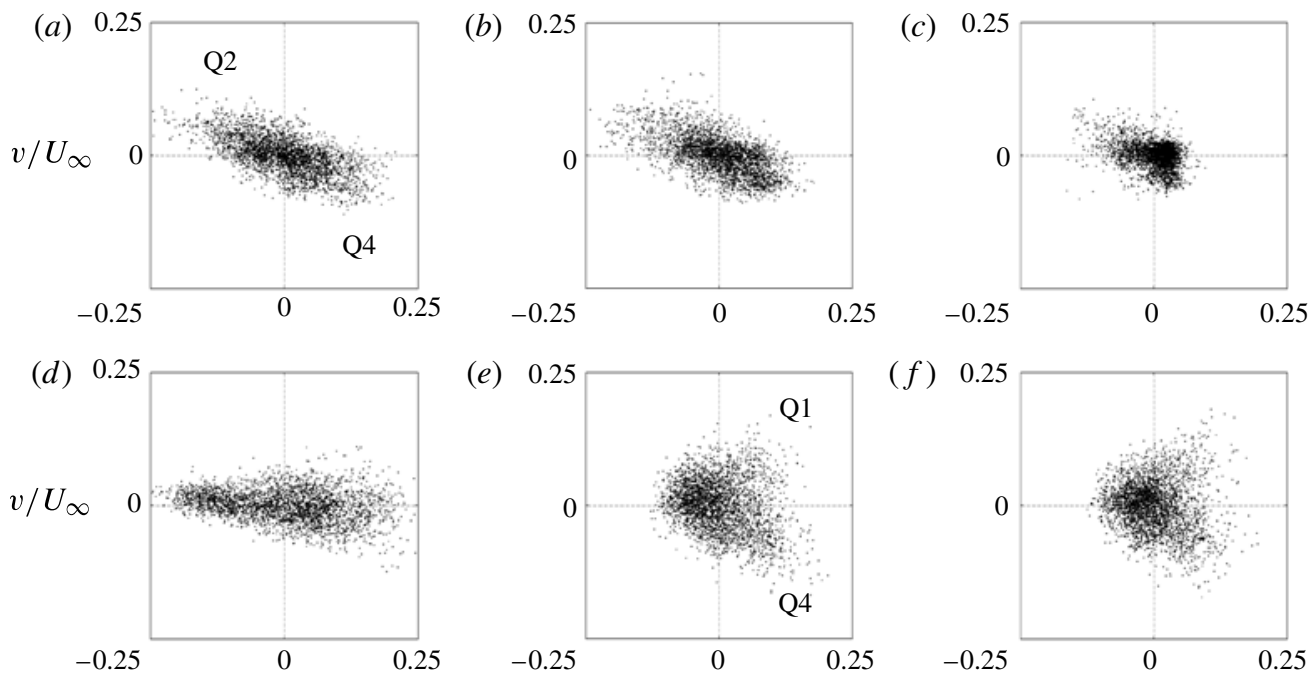

(e)
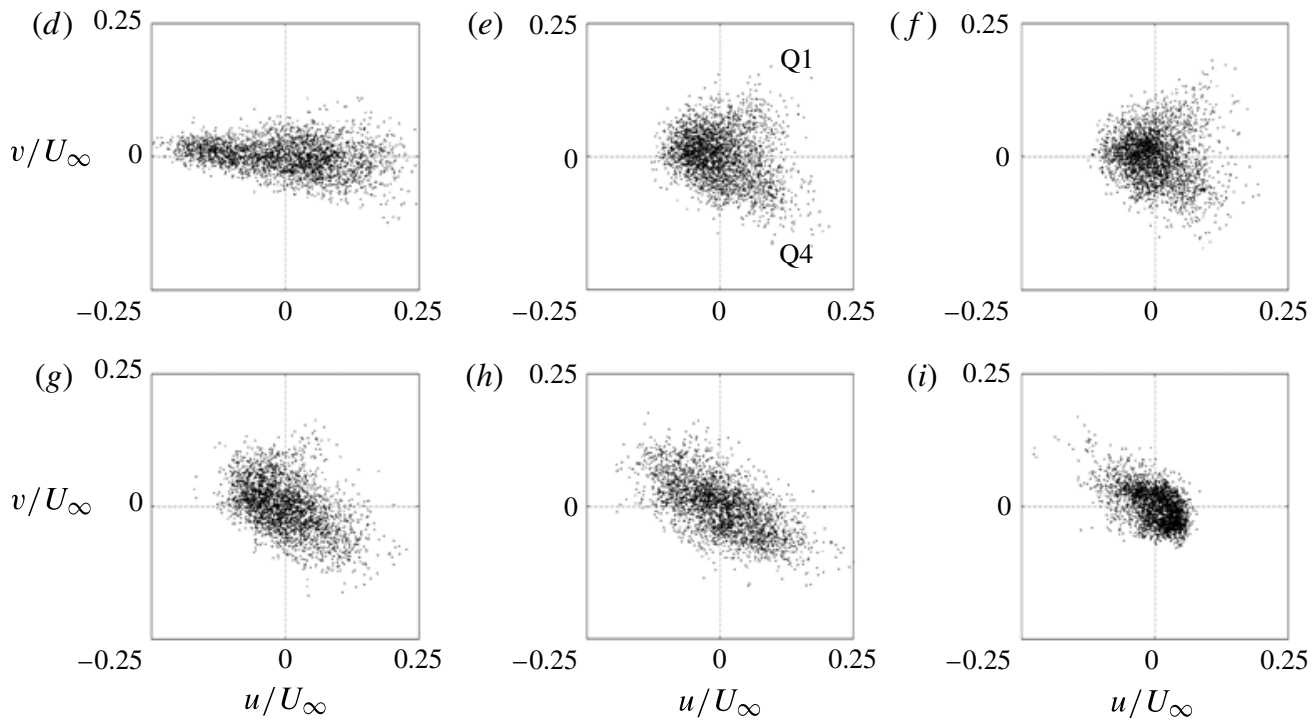

FIGURE 9. Quadrant analysis within the boundary layer and the wake: within the boundary layer at $s / \theta_{0}=-12$ and wall-normal locations of $(a) n / \theta_{0}=1.1$, (b) $n / \theta_{0}=2.9$ and (c) $n / \theta_{0}=4.7$; along the wake centreline at streamwise locations of $(d) x / \theta_{0}=0.3$, (e) $x / \theta_{0}=3.5$ and $(f) x / \theta_{0}=11.7$; and perpendicular to the wake centreline at $x / \theta_{0}=11.7$ and $(g) y / \theta_{0}=0.6$, (h) $y / \theta_{0}=2.9$ and $(i) y / \theta_{0}=4.7$. The dashed lines in $(a)$ and (e) emphasize the dominant quadrant event within the turbulent boundary layer and the subsequent wake, respectively.

properties along with the dynamic behaviour of these vortices is accomplished in $\S 4.2$ using the three-dimensional visualization of Tomo-PIV.

\section{Unsteady flow organization}

\subsection{Turbulent boundary layer}

The three-dimensional organization of the low- and high-speed streaks, ejection and sweep events, and the vortical structures within the boundary layer prior to the trailing edge is characterized using Tomo-PIV across $0.15-0.8 \delta_{99}$ of the boundary layer thickness (volume I of figure 2) as the necessary first step in studying their subsequent evolution into the wake region. Their organization is evaluated with respect to the typical structures observed in a zero-pressure-gradient boundary layer (Robin 1991).

The low- and high-speed streaks are identified as negative and positive $u$ perturbations of $u / U_{\infty}= \pm 0.1$ strength elongated alternately in the streamwise direction of figure $10(a)$. The low-speed streaks originally develop in the viscous 
sublayer $\left(<10^{+}\right)$and are lifted into the logarithmic region through the ejection process induced by the hairpins and the quasi-streamwise vortices (Smith \& Metzler 1983). They are observed to have an approximate width of $2.2 \theta_{0}\left(100^{+}\right)$at $n / \theta_{0}=2\left(100^{+}\right)$within the log layer and are separated by high-speed streaks of similar width agreeing with the $100^{+}$streak spacing recorded in the previous literature (Robin 1991). The streamwise extent of these structures cannot be evaluated with the current experimental configuration, as they have a meandering path that extends beyond the dimension of the FOV. The strength of the low- and high-speed streaks and its variation in the streamwise direction is investigated in $\S 5$. The visualization of figure 10(a) confirms the presence of both high- and low-speed streaks in the immediate upstream region of the trailing edge.

The majority of the vortical structures surround the low-speed streaks (figure 10b) due to their mutual interaction through the lift-up process. The region of the highspeed streaks is relatively depleted of significant vortical activity. The vortices partially surround the low-speed streaks, while the high-speed streaks are located on the two spanwise sides. The visualized vortex filaments are observed to have a radius of about $0.5 \theta_{0}\left(25^{+}\right)$based on an iso-surface of $Q=0.8 \times 10^{6} \mathrm{~s}^{-2}$. The full hairpin vortex specified in the magnified view of figure $10(b)$ has a leg spacing of about $3 \theta_{0}\left(140^{+}\right)$ surrounding the low-speed streak. A complete and undistorted hairpin vortex consisting of a spanwise portion (head) connected to two similar quasi-streamwise portions (legs) as identified here does not frequently form. However, its derivatives, such as streamwise or spanwise portions, are more commonly observed in a turbulent boundary layer (Guezennec, Piomelli \& Kim 1989). Samples of a leg and a cane (combination of a leg and a head) vortex are also identified in figure 10(b). The hairpin vortex and its derivatives may appear close together in a packet forming LSMs of a turbulent boundary layer (Adrian et al. 2000), which is also shown in figure $10(b)$.

The relation between the ejection and sweep events and the hairpin category of vortical structures is studied by identifying the ejection and sweep events in the magnified views of figure $10(b)$. The ejection events are identified here as iso-surfaces of $-u v / U_{\infty}^{2}=0.0026$ provided that the conditions $u<0$ and $v>0$ are respected. The opposing sweep event $(u<0$ and $v<0)$ is identified by the half-strength of $-u v / U_{\infty}^{2}=0.0013$, as they are weaker events. In a turbulent boundary layer, the ejections are stronger since they are generated by focused induction surrounded by a complete or portions of a hairpin vortex, while the sweep events tend to be weaker as they are unfocused induction on the outer boundaries of the hairpin vortices (Adrian 2007). The curvature around the head of the hairpin vortex aids in focusing the flow in the inboard region, making the ejection stronger than the sweep event. This feature also makes the ejection produced by a hairpin and a cane shape vortex stronger than that of an isolated leg.

A time sequence of the development of coherent structures prior to the trailing edge is shown in supplementary movie 1 available at journals.cambridge.org/flm. The movie is obtained by 10 times increase of the temporal resolution of the original measurement from 2700 to $27000 \mathrm{~Hz}$ using the advection equation (Scarano \& Moore 2011). The observed unsteady organization of coherent structures within the wallbounded region prior to the trailing edge adapts to the new slip-free permeable boundary condition along the wake centreline as investigated in the next subsection.

The kinematic features of the hairpin vortex are educed from an ensemble average of the detected structures using the pattern recognition algorithm described in $\$ 2.4$. The resulting vortex is described using an iso-surface of $Q=0.5 \times 10^{6} \mathrm{~s}^{-2}$ in 
(a)

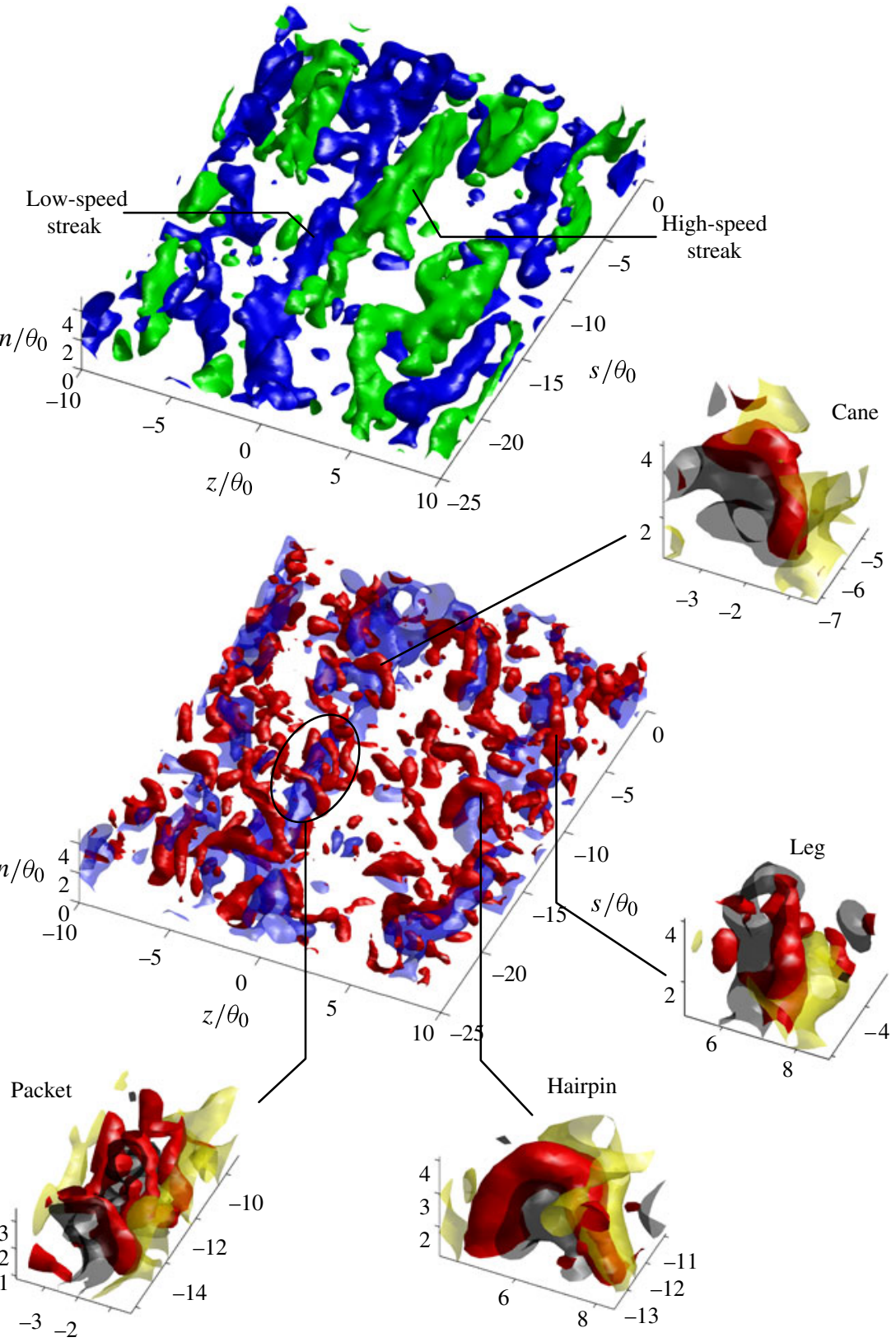

FIGURE 10. (Colour online) Instantaneous snapshot of three-dimensional organization of structures within the turbulent boundary layer prior to the trailing edge. (a) Organization of the low-speed (blue/dark grey) and high-speed (green/light grey) streaks visualized by iso-surfaces of $u / U_{\infty}= \pm 0.1$. (b) Interaction between the low-speed streaks (transparent blue/light grey) and vortical structures (red/dark grey) identified by $Q=0.8 \times 10^{6} \mathrm{~s}^{-2}$. Also shown are magnified details of a hairpin packet, a full hairpin, and leg and cane vortices. Ejection (grey/mid grey) and sweep (yellow/light grey) events are specified at $-u v / U_{\infty}^{2}=0.0026$ and 0.0013 , respectively. 
figure 11, demonstrating a hairpin shape vortex with leg spacing of $2.2 \theta_{0}\left(100^{+}\right)$ and height of $2 \theta_{0}\left(95^{+}\right)$. The vortex is also inclined at $60^{\circ}$ angle relative to the free stream direction, which is slightly higher than the characteristic $45^{\circ}$ inclination observed within zero-pressure-gradient boundary layers (Theodorsen 1952; Head \& Bandyopadhyay 1981) due to the flow curvature and the adverse pressure gradient in the vicinity of the trailing edge. The result of the ensemble averaging procedure exhibits slightly lower values of the peak vorticity as well as of $Q$. This is ascribed to macroscopic fluctuations (legs tilting or mutual shift), which cannot be accounted for by a simple alignment procedure. As a result, the display of the educed vortex is based on a value of $Q$ in figure 11 that is $60 \%$ less than that of figure 10 . The flow pattern induced by the hairpin vortex is illustrated by streamlines relative to the local advection velocity of the flow (Scarano 1999; Adrian et al. 2000). The ejection event is induced at the centroid of the vortex lifting up the low-speed streak and near-wall vorticity, resulting in more homogeneous distribution of vorticity (Adrian 2007). The centroid of the ejection event is close to the vortex head, demonstrating the effect of vortex curvature on the formation of a focused induction. The sweep event is observed in the outbound part of the hairpin vortex, which transports the high-speed fluid towards the wall, balancing the mass conservation in relation to the ejection event. The sweep event is visualized using $-u v / U_{\infty}^{2}=0.0002$, which is 20 times weaker than the typical value observed in ejections whereby $-u v / U_{\infty}^{2}=0.004$. The strength of the sweep event is also an order of magnitude less than that of the instantaneous samples of figure 10, which shows the high irregularity of the sweep region in the outbound part of the instantaneous hairpin vortices. A portion of the sweep event occurs at the outer sides of the two vortex legs coinciding with the neighbouring high-speed streaks. The rest of the sweep event occurs at the outbound region of the vortex head above the low-speed streak. The resulting alternate ejection and sweep feature along a low-speed streak is known as a variable-interval time-averaging (VITA) condition, and has been used to detect hairpin vortices from single-point measurement signals (Wallace, Brodkey \& Eckelmann 1997).

\subsection{Turbulent wake}

The Tomo-PIV visualization of the wake region (volume II of figure 2) demonstrates that the disappearance of the solid wall does not immediately affect the organization of the low- and high-speed streaks and they persist into the wake region as observed in figure 12. The low- and high-speed streaks exhibit an average thickness and spacing of $2.2 \theta_{0}\left(100^{+}\right)$similar to the conditions prior to the trailing edge. Similar to the upstream turbulent boundary layer, the streaks meander and extend beyond the measurement field in the streamwise direction. The hydrogen bubble visualization of Haji-Haidari \& Smith (1988) also confirmed the existence of low-speed streaks with a spacing of $100^{+}$in the immediate wake region. They have also observed that the low-speed streaks become weaker and less coherent with increasing distance from the trailing edge. This will be discussed further based on statistical evidence in $\S 5$, where the mitigation of the streaks is ascribed to the diminished viscous sublayer and the recovery process of the wake, which tends towards the homogeneous state of the far wake.

The vortical structures on the free stream side of the low- and high-speed streaks also show a gradual response to the disappearance of the solid wall and similar organization as the upstream turbulent boundary layer. The high-speed streak of figure 13(a) shows lower vortical activity relative to the low-speed streak at the free stream interface. There are only vortical structures on the two spanwise sides of 


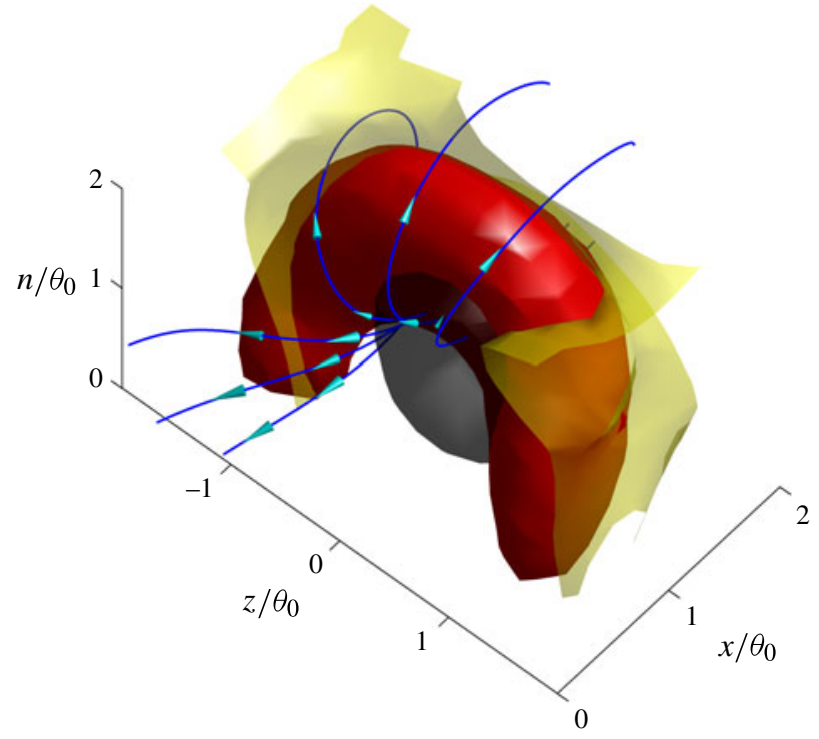

FIGURE 11. (Colour online) Ensemble-averaged hairpin vortex after pattern recognition analysis. The hairpin vortex is identified at $Q=0.5 \times 10^{6} \mathrm{~s}^{-2}$ (red/dark grey), while the ejection (grey/mid grey) and the sweep (yellow/light grey) events are identified by $-u v / U_{\infty}^{2}=0.0040$ and 0.0002 , respectively. The velocity streamlines are visualized relative to $U=0.78 U_{\infty}$ and $V=-0.04 U_{\infty}$.

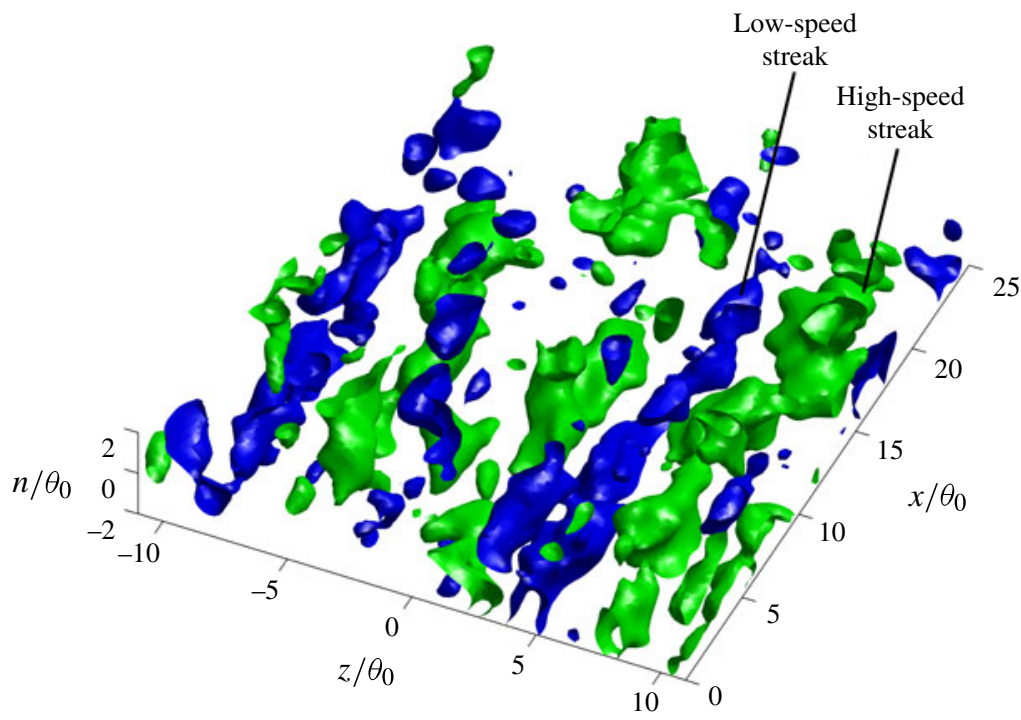

FIGURE 12. (Colour online) Organization of low-speed (blue/dark grey) and high-speed (green/light grey) streaks within the wake region $\left(u / U_{\infty}= \pm 0.1\right)$.

the high-speed streak as they are in contact with neighbouring low-speed streaks due to the alternate arrangement of the high- and low-speed streaks. On the other hand, the free stream side of the low-speed streaks of figure $13(b)$ is populated with hairpin structures consisting of spanwise vortices with $\omega_{z}<0$ connected to quasi-streamwise 
(a)

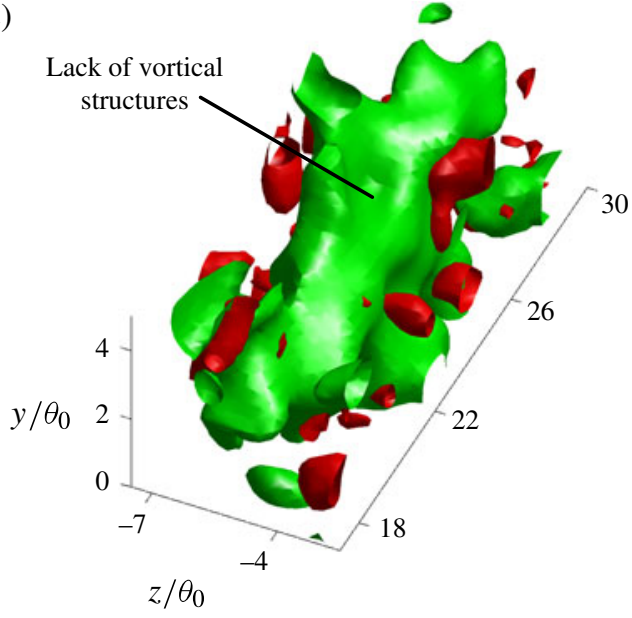

(b)

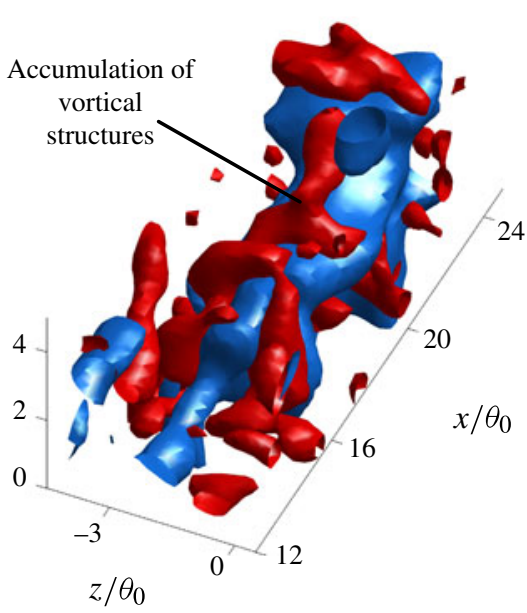

FIgURE 13. (Colour online) Vortical structures identified by $Q=0.5 \times 10^{6} \mathrm{~s}^{-2}$ (red/dark grey) on the free stream side of ( $a$ ) high-speed (green/light grey) and $(b)$ low-speed (blue/mid grey) streaks of the wake region of $u / U_{\infty}=0.1$ and -0.1 strength, respectively.

vortices at the sides of the low-speed streak inducing a focused ejection event. The accumulation of vortical activities around the low-speed streaks is due to the lift-up process of the coherent vorticity generated at the near-wall region by the ejection events. The high-speed streaks are formed by the sweeps of the high-velocity flow from the upper layers of the boundary layer that contains a low vorticity level.

The persistence of the hairpin vortices into the wake region agrees well with previous observations in the literature remarking upon the negligible change in the statistical and visual characteristics of the outer region of the turbulent boundary layer upon passage over the trailing edge (Robinson 1969; Haji-Haidari \& Smith 1988). In general, the outer layer of a turbulent boundary layer responds gradually to changes in the surface boundary condition such as roughness modification, as observed by Townsend (1965, 1966). The hairpin vortices are not significantly altered for some distance downstream and persist into the wake region as long as the low-speed streaks exist (see figure 13b). A reduction in the intensity of these vortical activities is observed as the low-speed streaks become weaker with increasing distance from the trailing edge. The ejection event induced by the hairpin vortices of the wake region erupts the low-speed fluid away from the centreline towards the free stream interface. The ejections promote the mixing process within the wake region and also widen the wake with increase of the streamwise direction. The larger hairpins form bulges at the wake interface and interact with the free stream.

The sudden disappearance of the solid wall adds another degree of interaction between the low- and high-speed streaks. Those arriving from the turbulent boundary layers of the two sides of the trailing edge can also interact and form vortical structures. The uncorrelated turbulent fluctuations of the two boundary layers before the trailing edge result in three possible combinations of high-high $(\mathrm{HH})$, low-low (LL) and low-high (LH) speed streaks in the $x y$ plane, as shown in figure 14. Other arrangements based on partial neighbouring can also occur, but they are less significant and may be regarded as combinations of the above. The streaks of LL and $\mathrm{HH}$ combinations have merged along the centreline and there is no distinguishable interface between them. Both of these combinations show least vortical activity along 
their interface, as they possess minimum velocity difference in this region. On the other hand, the LH arrangement shows significant vortical activity at the streak interface along the centreline. The nature of these vortical activities is scrutinized in figure 15 in which a three-dimensional representation of the high-speed streak and the surrounding vortical structures of the LH cross-section of figure 14(c) is illustrated.

It is observed that the spanwise rolls of $\omega_{z}$ vorticity at the wake centreline between the high- and low-speed streaks of figure 15 are further extended by quasi-streamwise vortices forming U-shaped structures. These vortical structures are introduced here as counter-hairpin vortices and are oriented in an opposite direction with respect to the hairpin vortices, since the spanwise part (vortex head) is at the low-velocity region of the mean velocity profile while the quasi-streamwise parts (the legs) are stretched away. The inclination of the legs to the mean flow in the $x y$ plane suggests that these structures are strain-dominated. The spanwise portion of counter-hairpins has the same vorticity direction as that of the hairpin vortices $\left(\omega_{z}<0\right)$, making it rather difficult to distinguish them with the pointwise or even planar measurements of the previous investigations. The visualization at the right-hand side of figure 15 shows that the counter-hairpin vortices induce a focused sweep event while a weak ejection region covers the outbound part of the vortices. Andreopoulos \& Bradshaw (1980) have previously observed occasional positive $u$ fluctuations at the edge of the inner wake and speculated those to be the result of the inward motion of the outer-region fluid towards the wake centreline. Later Haji-Haidari \& Smith (1988) compared this inward-moving fluid to sweep events of a turbulent boundary layer and associated them to streamwise vortices inducing non-periodic inrushes of the outer fluid towards the wake centreline. However, the spanwise portions of these vortices have escaped hydrogen bubble visualization and the complete vortex structure could not be identified. Furthermore, figure 14 shows that the inrushes of fluid towards the wake centreline cannot be assimilated to the sweep events of a turbulent boundary layer. Instead, the induction mechanism resembles that of ejection events, with proper inversion of properties, as they are both focused events. The sweep events generated by the counter-hairpin vortices act in the reverse direction of the ejection event induced by hairpin vortices but with similar strength. The other distinguishing feature of the counter-hairpins is that they surround the high-speed streaks, contrary to the hairpin vortices. The counter-hairpin vortices generate sweeps of the high-speed fluid within the wake towards the centreline, promoting the recovery of the wake deficit. The sweep events also result in bulges on the interface of low- and high-speed streaks along the wake centreline, which results in the wavy nature of the wake inner layer as characterized by Andreopoulos \& Bradshaw (1980).

The vortical structures within the wake region can be distinguished from those of the boundary layer by their accumulation around the high-speed streaks. This is observed in the instantaneous Tomo-PIV snapshot of figure 16 in which a significant number of vortices are observed partially wrapped around the high-speed streaks. Two samples of counter-hairpin vortices are also specified with a pronounced U-shaped structure, with the spanwise section close to the wake centreline. The magnified views also demonstrate the embedded sweep event in the centroid of these vortices, while the surrounding ejection event appears irregular and weak. Further scrutiny of the Tomo-PIV snapshots of the wake region shows that the probability of occurrence of a complete counter-hairpin as shown in figure 16 is relatively low. Similar to the hairpin vortices of a turbulent boundary layer, the derivatives such as cane or leg shape vortices are more frequent. Samples of full hairpins and the derivatives (leg and cane vortices) are shown in figure 17. Different vortical shapes can be caused as a result of 

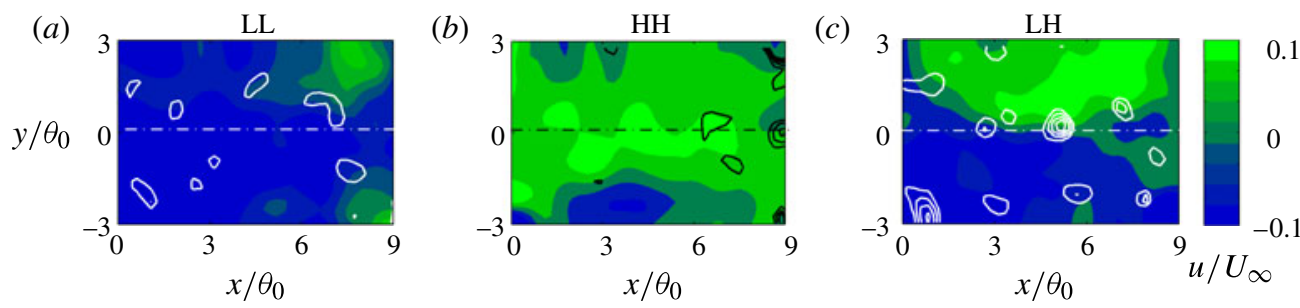

FIgURE 14. (Colour online) The vortical structures at the interface between three combinations of $(a)$ low-low (LL), $(b)$ high-high (HH) and $(c)$ low-high (LH) interfaces are considered. The solid contours show spanwise vortex cores identified by swirling strength $\lambda_{c i}$ (Tomkins \& Adrian 2003).
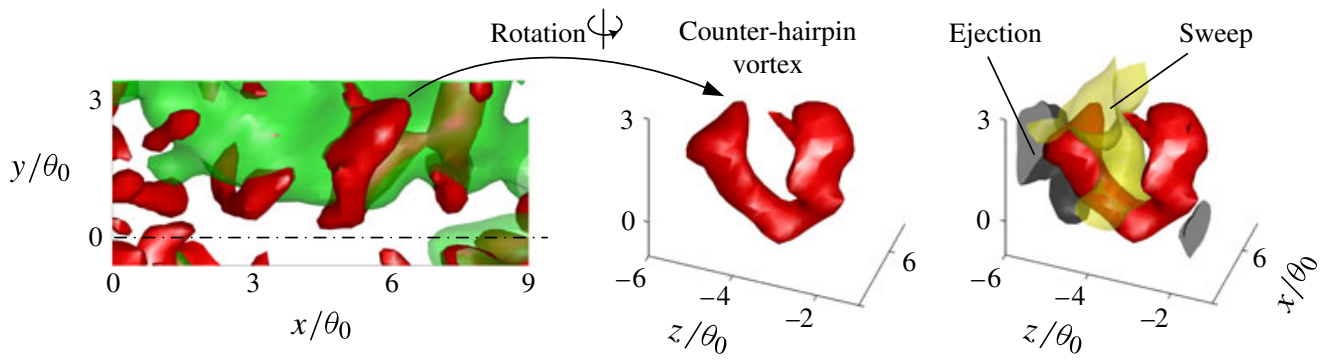

FIGURE 15. (Colour online) The three-dimensional image (left) shows the vortical structures (red, $Q=0.8 \times 10^{6} \mathrm{~s}^{-2}$ ) wrapped around the high-speed streak (green) of the LH interface of figure 14(c). A counter-hairpin is isolated from the group of vortical structures and visualized separately, with its corresponding sweep (yellow) and ejection (grey) regions identified by $-u v / U_{\infty}^{2}=0.01$ and $-u v / U_{\infty}^{2}=0.001$, respectively.

non-uniform stretching, partial tearing, cutting and connecting (Adrian 2007). However, these derivatives can be categorized as counter-hairpins as long as they contribute to the same transport phenomenon by sharing an induction of a strong focused sweep while locating on a high-speed streak. This is the case for complete hairpins and the canes, while the legs are shared in the spanwise interface of a low- and highspeed streak and can induce sweep or ejection of similar strength depending on their curvature. Therefore, the legs are shared between the hairpin and counter-hairpin signatures and promote spanwise mixing. The existence of different derivatives of the counter-hairpins makes their characterization difficult and necessitates a systematic eduction of their features using the described pattern recognition algorithm.

The ensemble-averaged counter-hairpin vortex is shown by the iso-surface of $Q=0.5 \times 10^{6} \mathrm{~s}^{-2}$ in figure 18. The analysis confirms the existence of counter-hairpin vortices in the wake region in a large realization set and demonstrates the mean kinematic features of the vortex structure. In general, the ensemble average of hairpins or horseshoe-like vortices can suffer due to distortion caused by unequal spanwise leg spacing, asymmetry in spanwise direction, unequal strength of the two legs, relative shift of the legs in the streamwise direction, and diffusion (Hayakawa \& Hussain 1989). When the above effects are important, the ensemble-averaged structure will be affected. In the present case, these effects are minimized (figure 18) by using a high threshold level in the pattern recognition algorithm, therefore rejecting the 


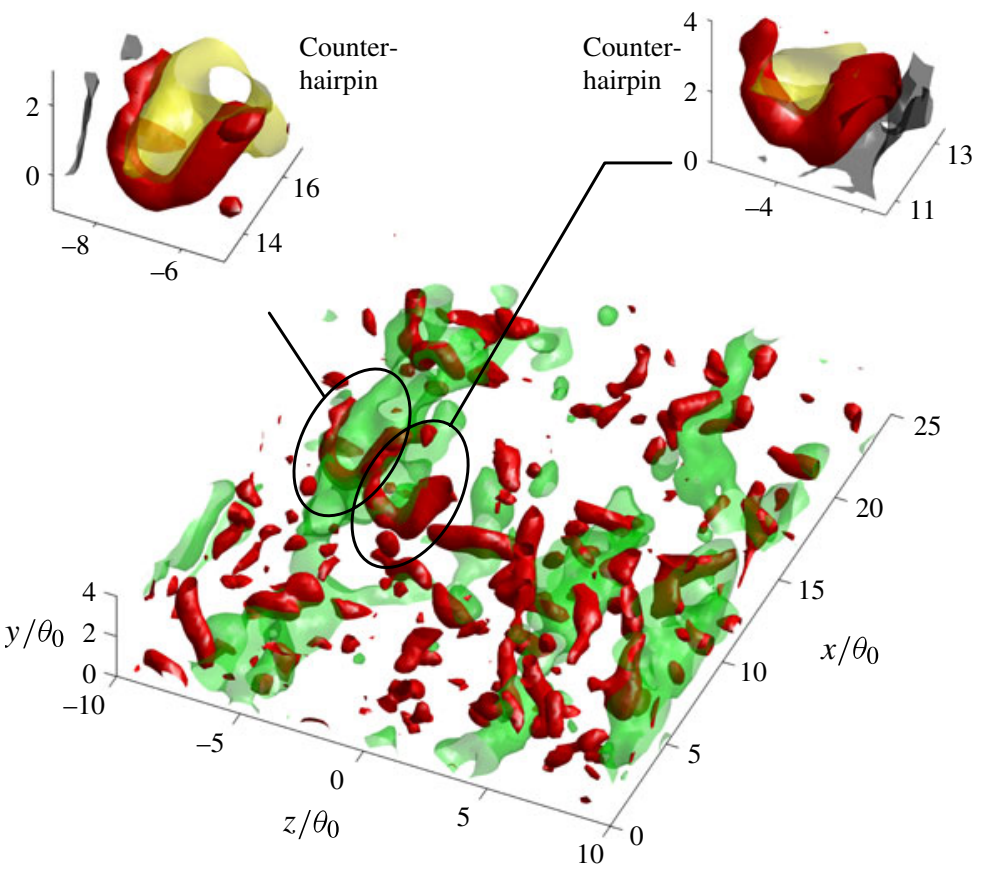

FIGURE 16. (Colour online) Instantaneous snapshot of three-dimensional organization of vortical structures (red/dark grey, $Q=0.8 \times 10^{6} \mathrm{~s}^{-2}$ ) and high-speed streaks (green/pale grey, $u / U_{\infty}=0.1$ ) within the wake region. The magnified views show two samples of counter-hairpin vortices along with the sweep (yellow/pale grey) and ejection (mid-grey) events identified at $-u v / U_{\infty}^{2}=0.003$ and 0.0015 levels, respectively. A time sequence of the development of coherent structures in the wake region is available in supplementary movie 2.

most distorted counter-hairpin structures. The average counter-hairpin has a height and leg spacing of $2 \theta_{0}\left(95^{+}\right)$. The dimensions are close to those of the average hairpin vortex of figure 11 because they are imposed by the width and height of the lowand high-speed streaks, which do not change considerably in the developing wake region. The inclination angle is $60^{\circ}$ with respect to the streamwise direction following a strain-dominated orientation. A focused sweep of the fluid is shown following the streamlines. The streamlines demonstrate higher curvature around the spanwise portion of the vortex due to the higher vorticity of this region. The sweep event is identified with an iso-surface of $-u v / U_{\infty}^{2}=0.004$ showing a focused sweep of high-speed flow at the centroid of the counter-hairpin vortex. The ejection event is visualized at 20 times lower strength $\left(-u v / U_{\infty}^{2}=0.0002\right)$ relative to the sweep event and covers the outbound region of the vortex.

\section{Spatio-temporal development of coherent structures}

The temporal description of the dynamical phenomena occurring in the vicinity of the trailing edge is obtained by time-resolved Tomo-PIV. The evolution of the low- and high-speed streaks and the hairpin structures is initially investigated. The formation of the counter-hairpin vortices is also discussed through the interaction of the turbulent streams from the two sides of the aerofoil. 
(a)

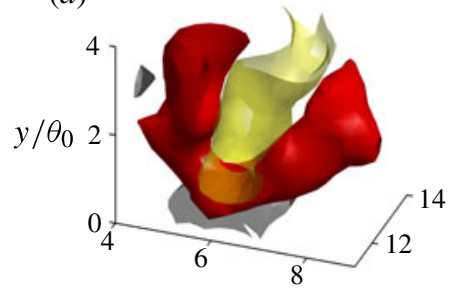

(d)

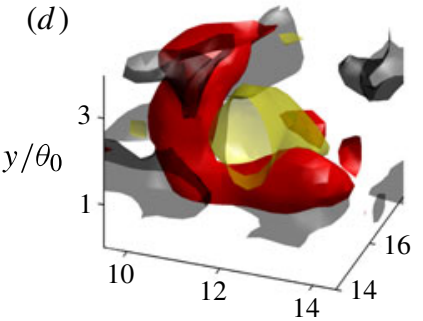

$(g)$

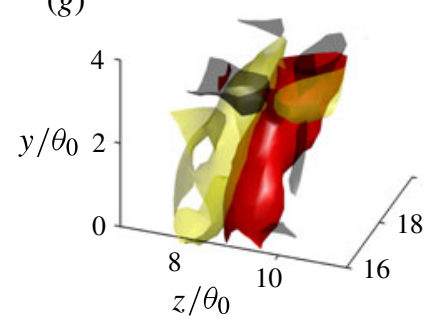

(b)

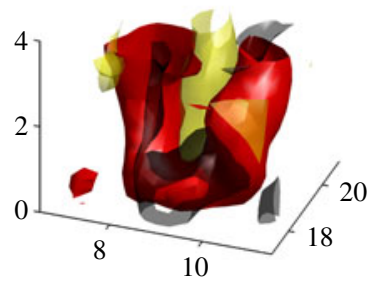

(e)

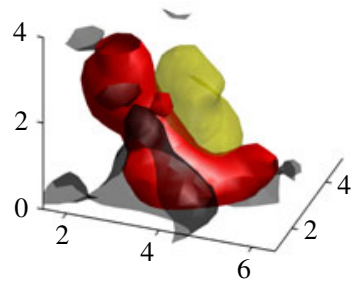

(h)

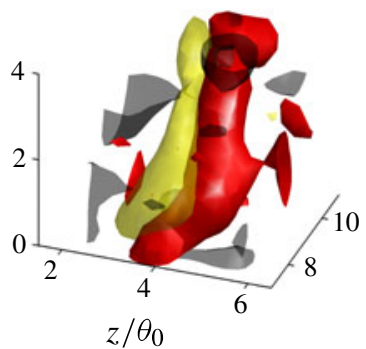

(c)

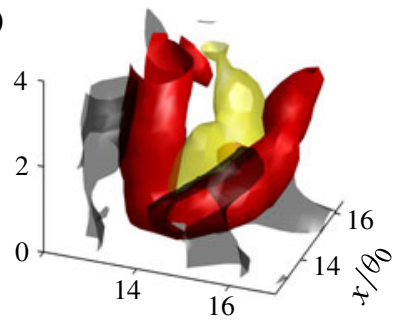

$(f)$

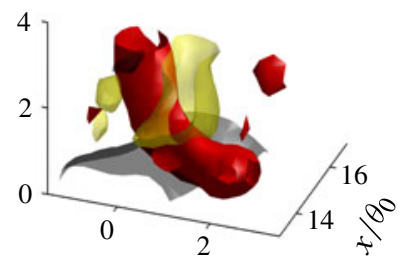

(i)

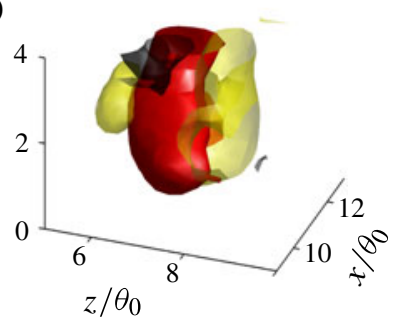

FIgURE 17. (Colour online) Derivatives of counter-hairpin vortices consisting of $(a-c)$ complete counter-hairpin, $(d-f)$ cane and $(g-i)$ leg vortices. The vortex structure (red/dark grey) is identified by $Q=0.8 \times 10^{6} \mathrm{~s}^{-2}$, while the sweep (yellow/pale grey) and ejection (mid-grey) are identified at $-u v / U_{\infty}^{2}=0.003$ and 0.0015 , respectively.

\subsection{Low- and high-speed streaks}

The variation in the strength of the low- and high-speed streaks in the vicinity of the trailing edge is ascribed to the interaction with the hairpins and counter-hairpins, which act oppositely to each other. The strength of the low- and high-speed streaks is evaluated by averaging the positive and negative $u$ fluctuations along the streamwise direction. A threshold of $u / U_{\infty}>0.05$ and $u / U_{\infty}<-0.05$ is selected to ensure that the averaging is conducted over coherent structures of the flow. The low-speed streaks are observed to be stronger than the high-speed streaks upstream of the trailing edge as observed in figure 19. An increase in the strength of both low- and high-speed streaks is observed as flow approaches the trailing edge, but this rate is slightly higher for the high-speed streaks. The intensification of the low-speed streaks is associated to higher lift-up rate of the viscous sublayer due to increase in the boundary layer thickness prior to the trailing edge. The strengthening of the low-speed streaks is accompanied by higher vortical activities and consequently the formation of strong high-speed streaks. It is also observed that the disappearance of the trailing edge immediately affects the strength of the low-speed streaks and their growth rate rapidly terminates at $x / \theta_{0}=0$. However, it requires a longer time till it considerably affects the organization of the structures in the wake region. A similar maximum in the growth rate of the high-speed streaks is observed after $5 \theta_{0}$ delay, indicating that they 


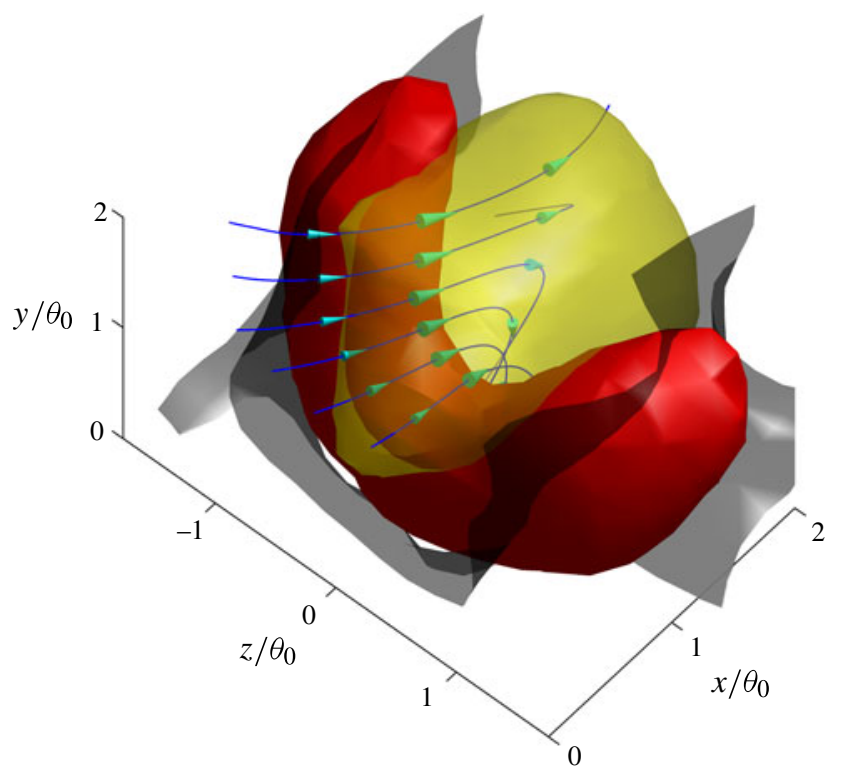

FIGURE 18. (Colour online) Ensemble-averaged counter-hairpin vortex obtained with pattern recognition. The vortex (red/dark grey) is identified at $Q=0.5 \times 10^{6} \mathrm{~s}^{-2}$, while the sweep (yellow/light grey) and the ejection (grey/mid grey) events are identified by $-u v / U_{\infty}^{2}=0.0002$ and 0.004 , respectively. The velocity streamlines are visualized relative to $U=0.66 U_{\infty}$.

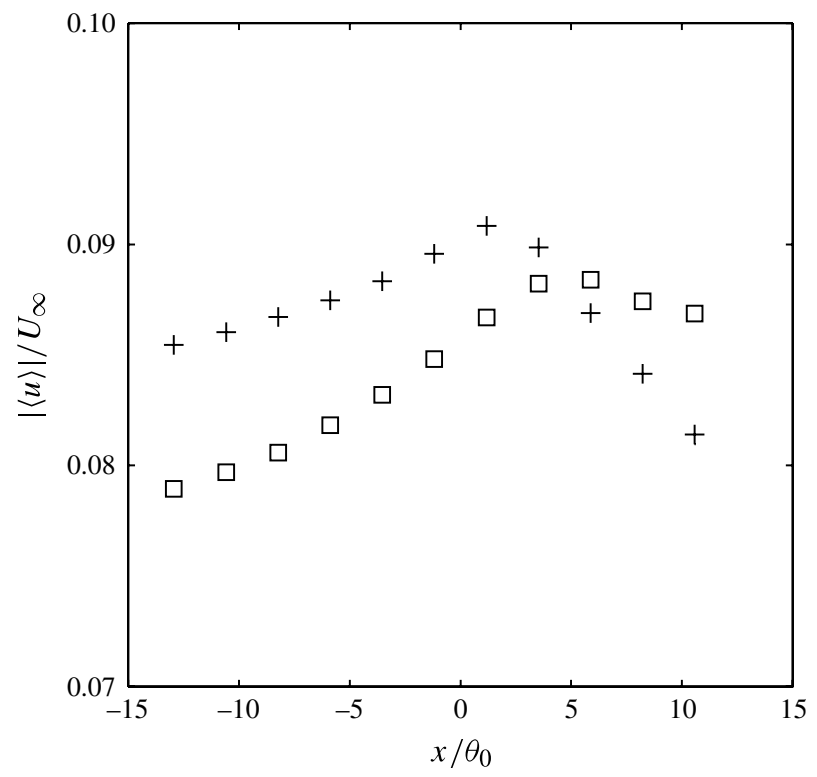

FIGURE 19. The strength of the low-speed streaks $(+)$ and the high-speed streaks $(\square)$ along the streamwise direction estimated by $u / U_{\infty}>0.05$ and $u / U_{\infty}<-0.05$ thresholds, respectively.

are indirectly influenced by the disappearance of the solid wall. After the trailing edge, the strength of the high-speed streaks is observed to decline at a lower rate in comparison to the low-speed streaks. The lower decay rate of the high-speed streaks is 

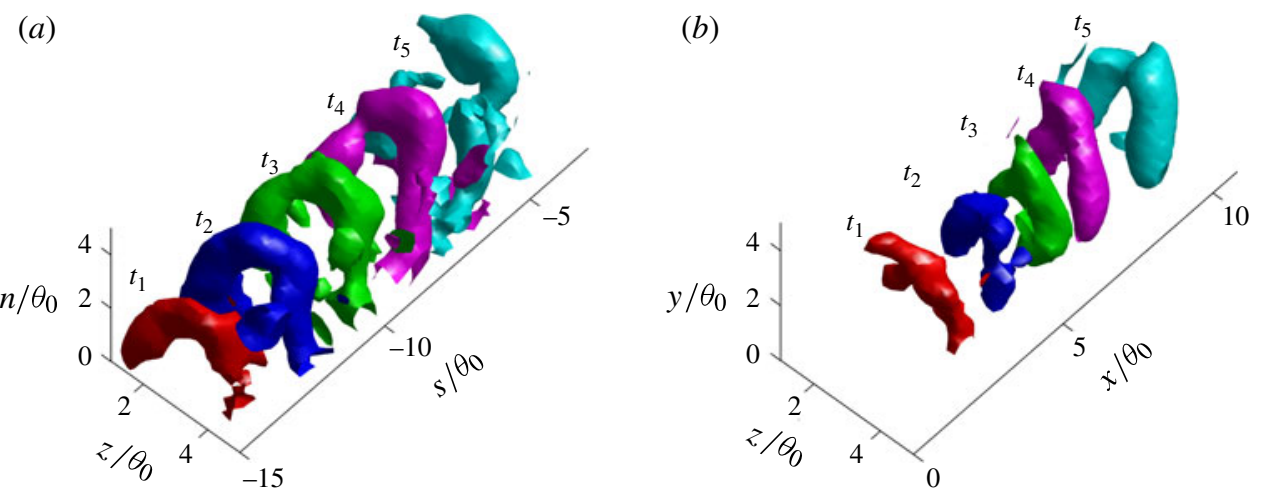

FIgURE 20. (Colour online) Time sequence of the evolution of a hairpin vortex initiated and terminated $(a)$ in the boundary layer prior to the trailing edge and $(b)$ in the wake region.

due to initiation of the counter-hairpin vortical activities immediately after the trailing edge opposing their decay by forming packets of sweep events promoting coherent regions of $u>0$.

\subsection{Hairpin vortices}

The transport of the hairpin vortices from the boundary layer into the wake region does not significantly affect the evolution of the hairpin vortices as shown in figure 20 . The left panel shows $1.5 \times 10^{-3} \mathrm{~s}$ of the life span for a hairpin vortex within the boundary layer prior to the trailing edge. At the first frame $\left(t_{1}\right)$, a cane vortex consisting of a spanwise portion (head) and a quasi-streamwise portion (leg) is observed. The vortex draws vorticity from the shear flow and grows as it moves downstream, forming a complete hairpin in the second frame $\left(t_{2}\right)$, which is $370 \mu \mathrm{s}$ later. In the next frames the vortex is further stretched, resulting in gradual rupture of the left leg. The onset of instability visualized at frame $t_{4}$ is unique, as the previous visualizations using Tomo-PIV were based on linear stochastic estimation and only the effect of diffusion is observed (Elsinga 2008). In the last frame $\left(t_{5}\right)$, the vortex is broken into smaller parts, which will be subject to further attenuation by diffusion. This growth mechanism of horse-shoe vortices by continuously drawing vorticity from the mean flow has already been proposed by Perry \& Chong (1982), which leads to a hierarchy of hairpin size and a logarithmic profile of the mean flow.

The vortex in the right-hand panel of figure 20 has initiated after the trailing edge in the wake region and grows within the visualized time span of $1.5 \times 10^{-3} \mathrm{~s}$. The persistence of this hairpin structure into the wake region is due to the continuation of the low-speed streaks in this region, as shown in figure 12. However, at the downstream edge of the measurement volume where the low-speed streaks are weaker, hairpin vortices are observed to occur less frequently. The investigation shows that the growth of the hairpin vortices occurs in the developing wake region similar to that of a turbulent boundary layer in spite of the ongoing mitigation process of the low-speed streaks.

\subsection{A generation mechanics for counter-hairpin vortices}

In the previous section, the counter-hairpin vortices have been detected along the wake centreline at the interface between a low- and a high-speed streak, suggesting that the formation mechanism is based on the existence of a sufficiently sheared region. 


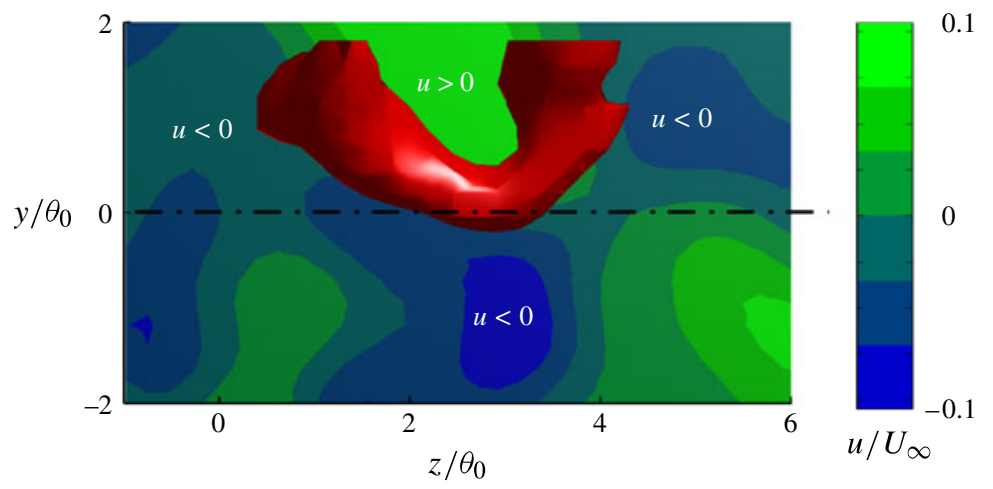

FIGURE 21. (Colour online) Cross-section of $u$ fluctuations across a counter-hairpin vortex (red/dark grey). The counter-hairpin partially surrounds a high-speed streak (green/light grey), while it is surrounded by low-speed streaks (blue/dark grey) from three sides.

The high-speed streak of the wake region, similar to those of the turbulent boundary layer, is adjacent to low-speed streaks in the spanwise direction, which ensures the existence of enough shear to supply the legs of the counter-hairpin vortices. However, the spanwise section of the counter-hairpin vortex also depends on the existence of a velocity difference at the wake centreline. Investigation of the Tomo-PIV snapshots shows that this condition of velocity difference can be met by the simultaneous arrival of a high- and a low-speed streak from the two sides of the trailing edge. The cross-sectional view of figure 21 shows the $u$ fluctuation in a $y z$ plane crossing through a counter-hairpin vortex in the wake region. The high-speed streak embedded in the centroid of the counter-hairpin vortex is in contact with a low-speed streak on the other side of the centreline, while it is also surrounded by two low-speed streaks on the spanwise sides. As a result, the contact with highly sheared regions from three sides promotes the formation of a counter-hairpin vortex involving intense vortex stretching, as detailed below.

In order to further evaluate the formation of the spanwise portion of the counterhairpin vortices as a result of shear along the wake centreline, the strength of the detected vortex cores is plotted versus instantaneous shear along the wake centreline. The vortex cores are identified using signed swirling strength $\lambda_{s}=\lambda_{c i}\left(\omega_{z} /\left|\omega_{z}\right|\right)$ following Tomkins \& Adrian (2003) to account also for the direction of rotation. The high shear at the centreline is caused by the simultaneous arrival of a low- and a high-speed streak from the two sides of the trailing edge. Therefore, the shear is evaluated by the velocity difference, $\Delta u$, averaged over two lines at $y / \theta_{0}= \pm 1$ distance from the centreline extending from $x / \theta_{0}=0$ to 12 . The velocity difference $\Delta u$ is considered as the velocity of the upper line $(y>0)$ minus that of the lower line $(y<0)$. The results are plotted in figure 21, confirming that the two adjacent low- and high-speed streaks on the opposite sides of the wake centreline cause the formation of vortex cores with rotation in the direction of the mean shear. Following the discussed counter-hairpin paradigm, these vortex cores are the spanwise portions of counter-hairpin vortices or their derivatives.

A time sequence of the formation mechanism of a counter-hairpin vortex is shown in figure 23. The first cross-section at $t_{0}$ corresponds to a location upstream of the trailing edge at $s / \theta_{0}=-5$ where two quasi-streamwise vortices (hairpin legs) are observed on the two sides of a high-speed streak. These quasi-streamwise vortices 


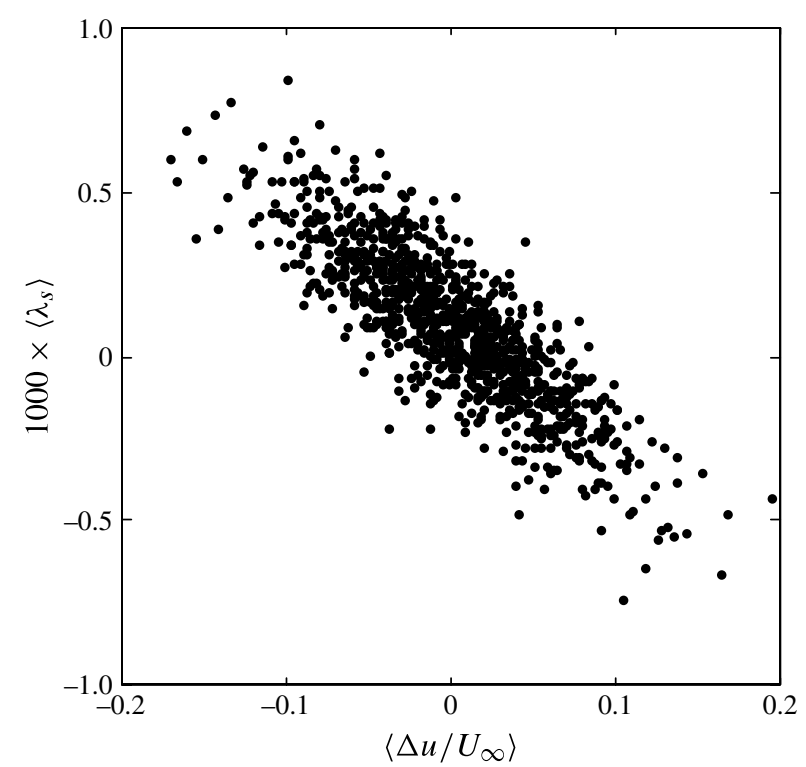

FIGURE 22. Vortex strength $\lambda_{s}$ versus mean shear rate along the wake centreline.

belong to the neighbouring low-speed streaks according to the hairpin vortex signature (Adrian et al. 2000). At $t=t_{0}+370 \mu$ s corresponding to $x / \theta_{0}=3$ after the trailing edge, the quasi-streamwise vortices start to connected by a spanwise portion (head of the counter-hairpin). A velocity difference along the centreline as a result of the occurrence of a low- and high-speed streak on the two sides of the centreline is required to form the spanwise vortex. At this moment, the vortex is highly distorted as the legs arrive at the trailing edge with slight delay and different orientations. The head and the two legs further unify at a downstream location of $x / \theta_{0}=12$ corresponding to $t=t_{0}+4 \times 370 \mu$ s and form a more regular counter-hairpin shape. Based on this evidence, the formation mechanism of counter-hairpin vortices is realized by connection between two quasi-streamwise vortices advected into the wake from the upstream turbulent boundary layer. The connection is a spanwise vortex in the vicinity of the centreline formed by the shear resulting from a low-speed flow on the other side of the wake centreline. It is also possible that the quasi-streamwise vortices initiate in the wake and later connect by a spanwise vortex.

The ensemble-averaged vortex of figure 18 provides the feasibility to study the rate of change of vorticity on a statistical basis. The rate of change of vorticity for a flow of constant density is expressed by the vorticity transport equation

$$
\frac{\mathrm{D} \omega}{\mathrm{D} t}=(\boldsymbol{\omega} \cdot \nabla) \boldsymbol{U}+v \nabla^{2} \boldsymbol{\omega},
$$

where $\boldsymbol{\omega}$ and $\boldsymbol{U}$ are the vorticity and velocity vectors in three-dimensional space. The first term on the right-hand side of this equation shows the rate of change of vorticity due to stretching and tilting of a vortex line. Following Kundu \& Cohen (2004), the tilting and stretching components can be decomposed by considering an intrinsic coordinate system $\boldsymbol{s}-\boldsymbol{n}$, where $\boldsymbol{s}$ is tangent to the vortex line and $\boldsymbol{n}$ points towards the velocity component normal to the vortex line, as shown in figure 24(a). This choice of coordinate system results in $\boldsymbol{\omega} \cdot \boldsymbol{i}_{n}=0$ and $\boldsymbol{\omega} \cdot \boldsymbol{i}_{s}=\omega$, where $\boldsymbol{i}_{s}$ and $\boldsymbol{i}_{n}$ are the unit vectors 


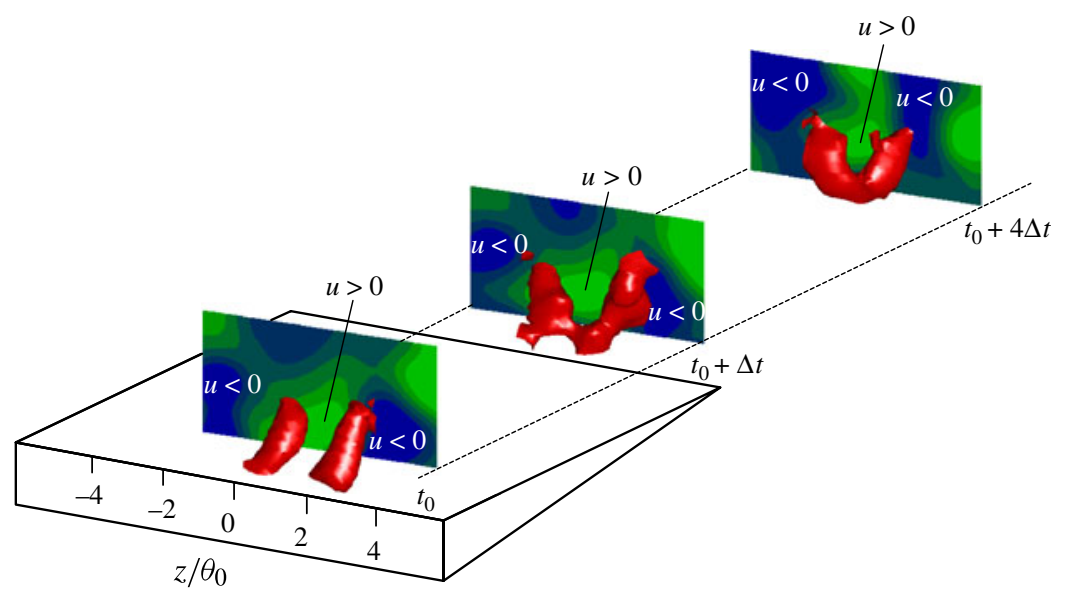

FIGURE 23. (Colour online) Time sequence of formation of a counter-hairpin vortex at $t_{0}$ (upstream of the trailing edge), $t_{0}+\Delta t$ (immediately after the trailing edge) and $t_{0}+4 \Delta t$ (downstream of the trailing edge). The visualization shows vortices identified by iso-surfaces of $Q=0.8 \times 10^{6} \mathrm{~s}^{-2}$ along with the spanwise wall-normal plane of $u$ fluctuations illustrating the cross-section of low- and high-speed streaks. The contour levels are similar to figure 21 .

in the tangential and normal directions, respectively. In this system of coordinates, the stretching-tilting term decomposes into

$$
(\boldsymbol{\omega} \cdot \nabla) \boldsymbol{U}=\left[\boldsymbol{\omega} \cdot\left(\boldsymbol{i}_{s} \frac{\partial}{\partial s}+\boldsymbol{i}_{n} \frac{\partial}{\partial n}\right)\right] \boldsymbol{U}=\omega\left(\frac{\partial U_{s}}{\partial s}+\frac{\partial U_{n}}{\partial s}\right),
$$

where $U_{s}$ and $U_{n}$ are the tangent and normal components of the velocity vector, respectively. The vorticity magnitude $(\omega)$ and the $\partial U_{s} / \partial s$ and $\partial U_{n} / \partial s$ terms are plotted in figure $24(b)$ along the specified vortex line of figure $24(a)$. The vorticity is slightly higher at the legs of the counter-hairpin vortex $\left(l / \theta_{0}= \pm 1.5\right)$, but its variation along the vortex line is rather small. The $\partial U_{s} / \partial s$ term shows intensification of vorticity due to positive stretching of the vortex line as a result of angular momentum conservation. The vortex stretching peaks around the head region of the counterhairpin $\left(l / \theta_{0}=0\right)$, causing a local vorticity increase and reduction of the vortex width. The latter can result in increased viscous diffusion $v \nabla^{2} \omega$. The tilting term $\partial U_{n} / \partial s$ is comparatively smaller and exhibits two peaks (positive and negative) at the neck regions $\left(l / \theta_{0}= \pm 0.5\right)$, which is known to be associated with the onset of vortex breakdown (Darmofal 1993). Also, the time sequence visualization of figure 20 suggests the onset of vortex breakdown around the neck regions, agreeing well with the position where the peak tilting motion is observed.

\section{Summary and discussion}

The wake of a sharp symmetric trailing edge is a mixing region between boundary layers arriving from the two sides of the trailing edge. The turbulent mechanism within the wake has similarities and differences with respect to its upstream boundary layer. The solid wall introduces a region of low velocity and high vorticity in a turbulent boundary layer, which is further transported away by the mixing process, and consequently boundary layer thickness increases. On the other hand, the turbulent mixing process within the wake region is redirected towards the recovery of the 

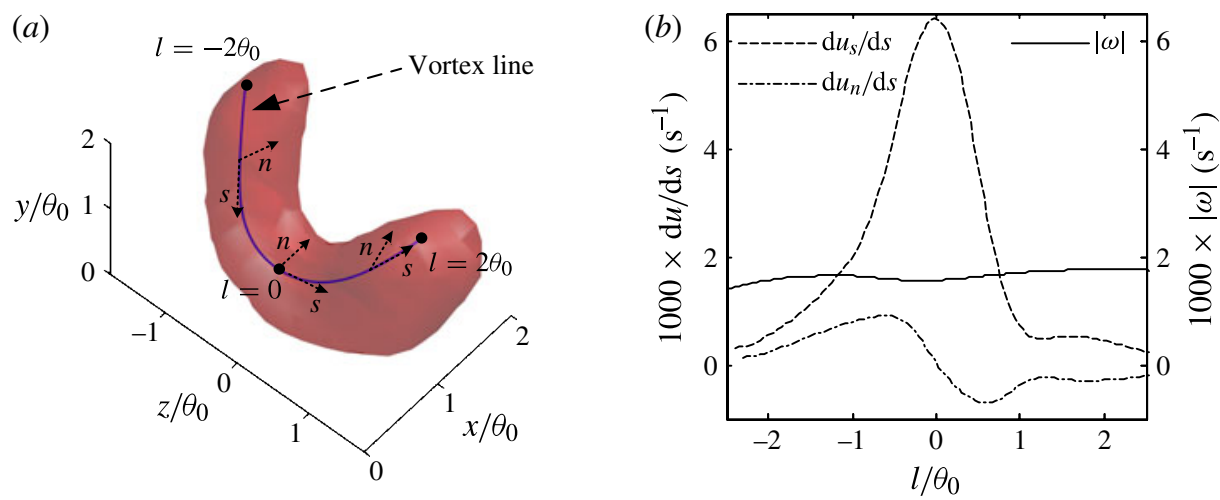

FIGURE 24. (Colour online) (a) A vortex line through the ensemble-averaged counter-hairpin vortex. (b) Vorticity, and tilting and stretching terms along the vortex line.

wake deficit (see figure $6 a$ ) via transport of high-velocity fluid towards the wake centreline (Chevray \& Kovasznay 1969). The change in the net direction of turbulent transport after the trailing edge was shown in the profiles of the triple products within the turbulent boundary layer and the subsequent wake in figure $8(a, b)$. This mixing process also results in gradual growth of the wake thickness (Andreopoulos \& Bradshaw 1980).

The low-curvature streamlines in the vicinity of a sharp trailing edge avoid the formation of large-scale structures within the wake region and the mixing process is conducted by small-scale vortices. This small-scale mixing of the inner layer has been described as consisting of inrushes of high-speed fluid from the outer layers towards the wake centreline followed by the eruption of low-speed flow away from the centreline (Haji-Haidari \& Smith 1988). The inrushes dominate the eruptions as the net momentum transport is towards the wake centreline. The quadrant analysis of figure $9(d-f)$ clearly demonstrated that, along the wake centreline, the sweeps are stronger than the ejections and result in higher $u v$ correlation. The previous investigations by Haji-Haidari \& Smith (1988) has conjectured that these events are similar to those of the turbulent boundary layer. However, the investigations of this work have shown that different vortical structures perform the so-called ejections away from the centreline and the inrushes (or sweeps) of high-speed flow towards the wake centreline.

The ejection events have been demonstrated to be performed in the developing wake region by a focused induction of low-speed flow away from the centreline through a hairpin-type vortex similar to a turbulent boundary layer. A schematic illustration of the hairpin vortices in the boundary layer and the wake is shown in figure 25 . The hairpin vortices persist in the wake region as long as the low-speed streaks continue to exist. The spanwise portion of the largest hairpin vortices forms the interface of the wake with the free stream, causing occasional eruption of low-speed fluid towards the free stream. These eruptions dominate the mixing process in the outer layer of the wake and result in gradual increase of the wake thickness. The hairpin vortex also generates a sweep events around itself; however, it is weak and does not make a significant contribution to the inrushes of high-speed flow towards the wake centreline.

Investigation of Tomo-PIV snapshots of the wake region revealed that the sweeps of fluid towards the centreline are generated by counter-hairpin vortices as shown in the conceptual scheme of figure 25 . These vortices are topologically and functionally 


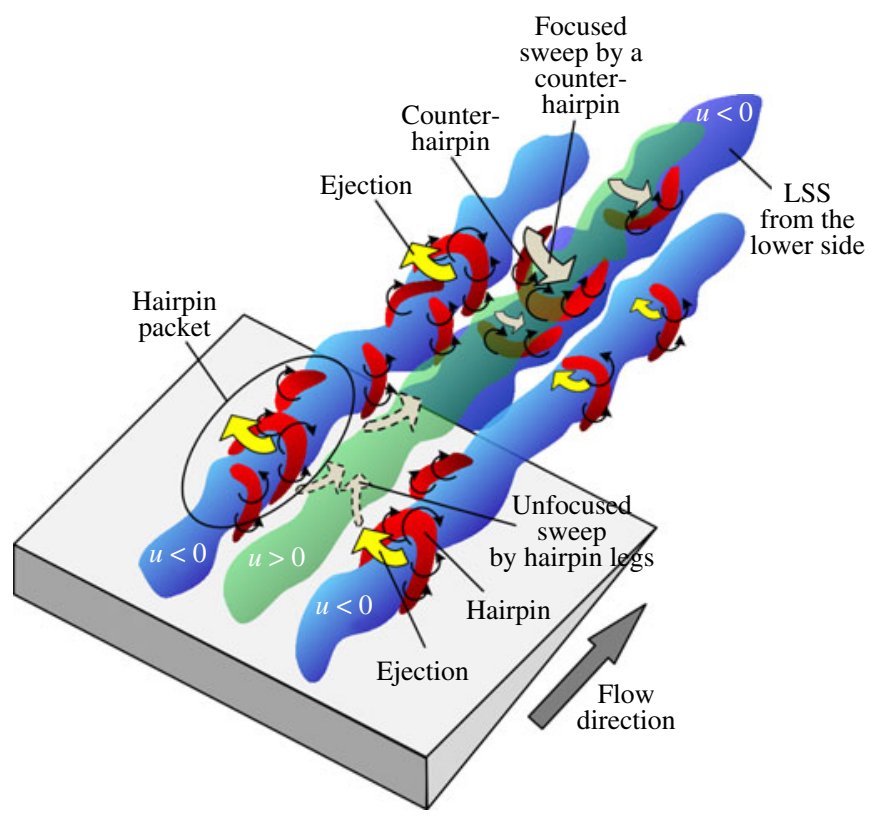

FIGURE 25. (Colour online) A conceptual scheme of the coherent structures across a sharp trailing edge showing the adaptation of the structures of the turbulent boundary layer into the new unbounded flow condition of the wake region. LSS = low-speed streak.

opposite to the hairpin vortices. They consist of a spanwise vortex (head) close to the wake centreline connected to two quasi-streamwise vortices (legs) that are stretched away at $60^{\circ}$ with respect to the streamwise direction as shown in figure 18 . The counter-hairpin vortices enclose the high-speed streaks and embed a focused sweep event transporting the high-speed fluid towards the wake centreline. The sweep events result in bulges of high-speed fluid intruding into the other side of the wake centreline, forming the wavy nature of the wake inner layer as observed by Andreopoulos \& Bradshaw (1980). There is also a weak ejection event at the outbound region of the counter-hairpin vortex.

The counter-hairpin vortices have not been observed in the turbulent boundary layer prior to the trailing edge and they are limited to the wake region (see figure 10 for the vortical structures within the boundary layer and figure 16 for the vortical structures of the wake region). The time-resolved Tomo-PIV snapshots demonstrate that the legs of the counter-hairpin vortices can originate upstream of the trailing edge where they interact with the low-speed streaks. The legs may connect after passing over the trailing edge by a spanwise vortex if there exists enough shear along the wake centreline. The presence of the high-shear region becomes viable by the disappearance of the wall and simultaneous arrival of a low- and a high-speed streak from the two sides of the trailing edge. This formation mechanism of counter-hairpin vortices was depicted in the time sequence of Tomo-PIV snapshots in figure 23. Therefore, the necessary shear to support a counter-hairpin vortex (enclose a high-speed streak) is provided by two low-speed streaks at the spanwise sides and a low-speed streak on the centreline side (see figure 21). A similar condition also applies to the hairpin vortex of a turbulent boundary layer, as the vortex encloses a low-speed streak and the high-shear regions at the three sides is provided by two high-speed streaks on the 
spanwise sides and a zone of higher momentum above it (Meinhart \& Adrian 1995; Adrian et al. 2000).

The appearance of the counter-hairpin vortices should be considered as an adaptation of the flow structures of the turbulent boundary layer to the unbounded condition of the wake. As observed in the quadrant analysis, both ejection and sweep events exist in the turbulent boundary layer due to the conservation of mass. However, the unfocused sweep events within the turbulent boundary layer that are induced by adjacent legs of the hairpin vortices become focused inductions in the wake region as a result of the formation of counter-hairpin vortices. Although there is an accumulation of vorticity at the lower side of the high-speed streaks within the turbulent boundary layer, the legs of the hairpins are not expected to connect as the existence of the wall and the high viscosity level prevents any roll-up of the vortex structures. After the trailing edge, the unbounded condition allows roll-up of vorticity and formation of spanwise vortex structures, which subsequently form the introduced counter-hairpins.

The introduced counter-hairpin paradigm along with the hairpin vortices and the lowand high-speed streaks satisfy the conditions previously mentioned in the introduction:

(i) The similarities in the statistical quantities such as mean and fluctuating velocity before and after the trailing edge are due to the persistence of similar structures, i.e. the low- and high-speed streaks and the hairpin vortices, into the wake region. In addition, the counter-hairpin vortices, which only belong to the wake region, have the same dimensions as the hairpin vortices.

(ii) The counter-hairpin vortices are the main contributor to the net transport of momentum towards the wake centreline and the recovery process of the wake deficit. Their activities distinguish the turbulent wake of a sharp trailing edge from the upstream turbulent boundary layer.

(iii) Although the ejection events and their associated $u v$ correlation gradually weakens by the mitigation of the low-speed streaks, the production of turbulence is kept at the same order of magnitude as that of the upstream turbulent boundary layer (see figure $7 a, b$ ) by addition of the sweep events in the wake region. The sweep events induced by the counter-hairpin vortices result in a strong $u v$ correlation and the production of turbulence in the wake region.

(iv) Both the hairpins and the counter-hairpin structures have a hierarchy of size and strength due to their growth mechanism. They grow by absorbing vorticity from the mean shear. The size of these vortices can extend as large as the wake half-width, which explains the existence of self-similar structures with increase of length scale along the wake centreline. As the wake grows, both the largest hairpin and the counter-hairpin vortices scale with the wake half-thickness and result in the logarithmic behaviour observed in figure $6(b)$.

\section{Conclusion}

An experimental investigation has been performed using 2C-PIV and Tomo-PIV in order to study the evolution of the unsteady organization of coherent structures of a turbulent boundary layer as they travel over the sharp trailing edge of a NACA0012 aerofoil. A pattern recognition algorithm is also applied to establish the kinematic features of the vortical structures based on ensemble averages.

Investigation of turbulent transport demonstrated similar net direction of transport for the turbulent boundary layer and the outer wake. In both regions, ejection events are dominant and transport low-speed fluid towards the free stream. The solid wall 
prior to the trailing edge continuously supplies the ejection events with low-speed flow, while immediately after the trailing edge the low-speed streaks start to deteriorate with the disappearance of the viscous sublayer. The weakening of the low-speed streaks gradually affects the high-speed streaks and they also start to weaken at about $5 \theta_{0}$ downstream of the trailing edge. The hairpin vortices that induce the ejection events continue to persist into the developing wake region, contributing to the mixing process and widening of the wake thickness.

The inner wake showed an opposite direction of net transport of turbulent fluctuations in comparison to the upstream boundary layer and the outer wake. The analysis of the triple products of turbulent fluctuations and the quadrant splitting of turbulent fluctuations confirmed that the net direction of transport of momentum and turbulent kinetic energy follows the sweep events towards the wake centreline. TomoPIV visualization of the wake region revealed that the sweep events are generated by vortices named here as counter-hairpin vortices. The counter-hairpin vortices are topologically similar to the hairpins but with an opposite orientation and function. The spanwise portion is located close to the wake centreline and the two attached quasistreamwise vortices are stretched outwards with $60^{\circ}$ angle with respect to the free stream direction. The counter-hairpin vortices partially surround the high-speed streaks, in contrast to the hairpin vortices that surround the low-speed streaks in a turbulent boundary layer. The centroid of a counter-hairpin vortex embeds a concentrated region of a sweep event, which transports the high-speed fluid and turbulent kinetic energy towards the wake centreline, promoting recovery of the wake deficit.

The formation mechanism of the counter-hairpin vortices has also been scrutinized using time-resolved Tomo-PIV visualizations. The investigations showed that the counter-hairpin vortices are formed as a result of the formation of spanwise vortices along the wake centreline upon disappearance of the solid wall. The sudden vanishing of the wall allows interaction of low- and high-speed streaks emerging from the two sides of the trailing edge, forming regions of high shear if a low- and high-speed streak appear on the two sides of the wake centreline.

\section{Acknowledgements}

This work was conducted as a part of the FLOVIST project (Flow Visualization Inspired Aeroacoustics with Time Resolved Tomographic Particle Image Velocimetry), funded by the European Research Council (ERC), grant no 202887.

Supplementary movies are available at journals.cambridge.org/flm.

\section{REFERENCES}

Adrian, R. J. 2007 Hairpin vortex organization in wall turbulence. Phys. Fluids 19, 041301.

ADRIAN, R. J. \& LIU, Z.-C. 2002 Observation of vortex packets in direct numerical simulation of fully turbulent channel flow. J Vis. 5, 9-19.

Adrian, R. J., Meinhart, C. D. \& Tomkins, C. D. 2000 Vortex organization in the outer region of the turbulent boundary layer. J. Fluid Mech. 422, 1-54.

ANDREOPOUlos, J. \& BRADShaW, P. 1980 Measurements of interacting turbulent shear layers in the near wake of a flat plate. J. Fluid Mech. 100, 639-668.

BLACKWELDER, R. F. \& ECKELMANN, H. 1979 Streamwise vortices associated with the bursting phenomenon. J. Fluid Mech. 94, 577-594.

BlAKE, W. K. 1986 Mechanics of Flow Induced Sound and Vibration, vols. I and II. Academic. 
Bogucz, E. A. \& WALker, J. D. A. 1988 The turbulent near wake at a sharp trailing-edge. J. Fluid Mech. 196, 555-584.

BRooks, T. F. \& Hodgson, T. H. 1981 Trailing edge noise prediction from measured surface pressures. J. Sound Vib. 78, 69-117.

Brooks, T. F., Pope, D. S. \& Marcolini, M. A. 1989 Aerofoil Self-Noise and Production. 1218. NASA Reference Publication.

Brouwer, H. H. \& Rienstra, S. W. 2008 Aeroacoustics research in Europe: the CEAS-ASC Report on 2007 highlights. J. Sound Vib. 318, 625-654.

CARlier, J. \& Stanislas, M. 2005 Experimental study of eddy structures in a turbulent boundary layer using particle image velocimetry. J. Fluid Mech. 535, 143-188.

Chevray, R. \& Kovasznay, L. S. G. 1969 Turbulence measurements in the wake of a thin flat plate. AIAA J. 8, 1641-1643.

Corino, E. R. \& Brodkey, R. S. 1969 A visual investigation of the wall region in turbulent flow. J. Fluid Mech. 37, 1-30.

DARMOFAL, D. L. 1993 The role of vorticity dynamics in vortex breakdown. AIAA 24th Fluid Dynamics Conference, Orlando, FL, AIAA 93-3036.

DEAN, R. B. \& BRADShAW, P. 1976 Measurements of interacting turbulent shear layers in a duct. J. Fluid Mech. 78, 641-676.

ElsingA, G. E. 2008 Tomographic particle image velocimetry and its application to turbulent boundary layers, PhD thesis, Delft University of Technology.

Elsinga, G. E., Adrian, R. J., Van Oudheusden, B. W. \& Scarano, F. 2010 Three-dimensional vortex organization in a high-Reynolds-number supersonic turbulent boundary layer. J. Fluid Mech. 644, 35-60.

Elsinga, G. E., Scarano, F., Wieneke, B. \& Van Oudheusden, B. W. 2006 Tomographic particle image velocimetry. Exp. Fluids 41, 933-947.

Ferré, J. A. \& Giralt, F. 1989 Pattern-recognition analysis of the velocity field in plane turbulent wakes. J. Fluid Mech. 198, 27-64.

Ffowcs Williams, J. E. \& Hall, L. H. 1970 Aerodynamic sound generation by turbulent flow in the vicinity of a scattering half plane. J. Fluid Mech. 40, 657-670.

Finez, A., Jondeau, E., Roger, M. \& Jacob, M. C. 2010 Broadband noise reduction with trailing edge brushes. 16th AIAA/CEAS Aeroacoustics Conference, Stockholm, Sweden, AIAA 2010-3980.

Ganapathisubramani, B., Longmire, E. \& Marusic, I. 2003 Characteristics of vortex packets in turbulent boundary layers. J. Fluid Mech. 478, 35-46.

GhaEmi, S. \& SCARANO, F. 2010 Multi-pass light amplification for tomographic particle image velocimetry applications. Meas. Sci. Technol. 22, 5.

Guala, M., Hommema, S. \& Adrian, R. 2006 Large scale and very large scale motions in turbulent pipe flows. J. Fluid Mech. 554, 521-542.

Guezennec, Y. G., Piomelli, U. \& KiM, J. 1989 On the shape and dynamics of wall structures in turbulent channel flow. Phys. Fluids A 1, 764-766.

Haji-Haidari, A. \& SMith, C. R. 1988 Development of the turbulent near wake of a tapered thick flat plate. J. Fluid Mech. 189, 135-163.

Hamelin, J. \& Alving, A. E. 1996 A low-shear turbulent boundary layer. Phys. Fluids 8 (3), 789-804.

Hayakawa, M. \& Hussain, F. 1989 Three-dimensionality of organized structures in a plane turbulent wake. J. Fluid Mech. 206, 375-404.

HAYAKAWA, M. \& IIDA, S. I. 1992 Behavior of turbulence in the near wake of a thin flat plate at low Reynolds numbers. Phys. Fluids A 4 (10), 2282-2291.

HeAd, M. R. \& BAndyopadhyay, P. 1981 New aspects of turbulent boundary-layer structure. J. Fluid Mech. 107, 297-338.

HebBAR, K. S. 1986 Mean and turbulence measurements in the boundary layer and wake of a symmetric aerofoil. Exp. Fluids 4, 214-222.

Herman, G. T. \& Lent, A. 1976 Iterative reconstruction algorithms. Comput. Biol. Med. 6, 273-294.

Herpin, S., Wong, C. Y., Stanislas, M. \& Soria, J. 2008 Stereoscopic PIV measurements of a turbulent boundary layer with a large spatial dynamic range. Exp. Fluids 45, 745-763. 
Humble, R. A., Elsinga, G. E., Scarano, F. \& Van Oudheusden, B. W. 2009 Three-dimensional unsteady flow organization of a shock wave/turbulent boundary layer interaction. J. Fluid Mech. 622, 33-62.

Hunt, J. C. R., Wray, A. A. \& Moin, P. 1988 Eddies, stream, and convergence zones in turbulent flows. Research Report CTR-S88, pp. 193-208. Center for Turbulence.

Hussain, A. K. M. F. 1986 Coherent structures and turbulence. J. Fluid Mech. 173, 303-356.

Hussain, F. \& HAYAKAWA, M. 1987 Education of large-scale organized structures in a turbulent plane wake. J. Fluid Mech. 180, 193-229.

Hutchins, N. \& MARUSIC, I. 2007 Evidence of very long meandering features in the logarithmic region of turbulent boundary layers. J. Fluid Mech. 579, 1-28.

Kline, S. J., Reynolds, W. C., Schraub, F. A. \& Runstadler, P. W. 1967 The structure of turbulent boundary layers. J. Fluid Mech. 30, 741-773.

Kundu, P. K. \& Cohen, I. M. 2004 Fluid Mechanics, 3rd edn. Elsevier.

Marsden, A. L., Wang, M., Dennis, J. E. \& Moin, P. 2007 Trailing-edge noise reduction using derivative-free optimization and large-eddy simulation. J. Fluid Mech. 572, 13-36.

Meinhart, C. D. \& AdRiAn, R. J. 1995 On the existence of uniform momentum zones in a turbulent boundary layer. Phys. Fluids 7 (4), 694-696.

Meinhart, C. D., Wereley, S. T. \& Santiago, J. G. 2000 A PIV algorithm for estimating time-averaged velocity fields. Trans. ASME: J. Fluids Engng 122, 285.

MiAU, J. \& KARLSSON, S. K. F. 1987 Flow structures in the developing region of a symmetric wake and an unsymmetric wake. Phys. Fluids 30 (8), 2389-2399.

MORRIS, S. C. \& FosS, J. F. 2003 Boundary layer to shear layer - the transitional region. J. Fluid Mech. 494, 187-221.

NAKAYAmA, A. \& LiU, B. 1990 The turbulent near wake of a flat plate at low Reynolds number. J. Fluid Mech. 217, 93-114.

NAsh, E. C., Lowson, M. V. \& MCAlpine, A. 1999 Boundary-layer instability noise of aerofoils. J. Fluid Mech. 382, 27-61.

Perry, A. E. \& Chong, M. S. 1982 On the mechanism of wall turbulence. J. Fluid Mech. 119, 173-217.

Pope, S. B. \& Whitelaw, J. H. 1976 The calculation of near-wake flows. J. Fluid Mech. 73, 9-32.

Ramaprian, B. R., PATel, V. C. \& SASTRY, M. S. 1982 The symmetric turbulent wake of a flat plate. AIAA J. 20 (9), 1228-1235.

Robin, S. K. 1991 Coherent motions in the turbulent boundary layer. Annu. Rev. Fluid Mech. 23, 601-639.

Robinson, J. L. 1969 Similarity solutions in several turbulent shear flows. Report 1242. National Physical Laboratory, Teddington, UK.

SAgrado, A. G., Hynes, T. \& Hodson, H. 2006 Experimental investigation into trailing edge noise sources. AIAA Paper 2006-2476.

SANDBERG, R. D. \& SANDHAM, N. D. 2008 Direct numerical simulation of turbulent flow past a trailing edge and the associated noise generation. J. Fluid Mech. 596, 353-385.

Scarano, F., Benocci, C. \& Riethmuller, M. L. 1999 Pattern recognition analysis of the turbulent flow past a backward facing step. Phys. Fluids 11 (12), 3808-3818.

SCARANO, F. \& MoORE, P. 2011 An advection-based model to increase the temporal resolution of PIV time series. Exp. Fluids doi:10.1007/s00348-011-1158-3.

SCARAno, F. \& Poelma, C. 2009 Three-dimensional vorticity patterns of cylinder wakes. Exp. Fluids 47, 69-83.

SCARAno, F. \& Reithmuller, M. L. 2000 Advances in iterative multigrid PIV image processing. Exp. Fluids 29, 51-60.

Schoppa, W. \& Hussain, F. 2000 Coherent structure dynamics in near-wall turbulence. Fluid Dyn. Res. 26, 119-139.

Schröder, A., Geisler, R., Elsinga, G. E., Scarano, F \& Dierksheide, U. 2008 Investigation of a turbulent spot and tripped turbulent boundary layer flow using time-resolved tomographic PIV. Exp. Fluids 44, 305-316. 
ScotT, C. M. \& Foss, J. F. 2003 Turbulent boundary layer to single-stream shear layer: the transition region. J. Fluid Mech. 494, 187-221.

Sharland, I. J. 1964 Sources of noise in axial flow fans. J. Sound Vib. 1 (3), 302-322.

Smith, C. R. \& Metzler, S. P. 1983 The characteristics of low-speed streaks in the near-wall region of a turbulent boundary layer. J. Fluid Mech. 129, 27-54.

Soloff, S. M., Adrian, R. J. \& LiU, Z. C. 1997 Distortion compensation for generalized stereoscopic particle image velocimetry. Meas. Sci. Technol. 8, 1441-1454.

SPALART, P. 1988 Direct simulation of a turbulent boundary layer up to $\operatorname{Re}_{\theta}=1410$. J. Fluid Mech. 187, 61-98.

Stanislas, M., Perret, L. \& Foucaut, J.-M. 2008 Vortical structures in the turbulent boundary layer: a possible route to a universal representation. J. Fluid Mech. 602, 327-382.

TAM, C. K. W. 1974 Discrete tones of isolated aerofoils. J. Acoust. Soc. Am. 55, 1173-1177.

TARDU, S. 1995 Characteristics of single and clusters of bursting events in the inner layer, Part 1: VITA events. Exp. Fluids 20, 112.

Theodorsen, T. 1952 Mechanism of turbulence. In Proceedings of the 2nd Midwestern Conference on Fluid Mechanics, Ohio State University, Columbus, OH, pp. 1-19.

TOMKIns, C. D. \& ADRIAN, R. J. 2003 Spanwise structure and scale growth in turbulent boundary layers. J. Fluid Mech. 490, 37-74.

Townsend, A. A. 1965 Self-preserving flow inside a turbulent boundary layer. J. Fluid Mech. 22, 773-797.

Townsend, A. A. 1966 The flow in a turbulent boundary layer after a change in surface roughness. J. Fluid Mech. 26, 255-266.

TUMMERS, M. J. 1999 Investigation of a turbulent wake in an adverse pressure gradient using laser Doppler anemometry, $\mathrm{PhD}$ thesis, Faculty of Aerospace Engineering, Delft University of Technology, The Netherlands.

Wallace, J. M., Brodkey, R. S. \& EckelmanN, H. 1997 Pattern-recognized structures in bounded turbulent shear flows. J. Fluid Mech. 83, 673-693.

Wallace, J. M., Eckelmann, H. \& Brodkey, R. S. 1972 The wall region in turbulent shear flow. J. Fluid Mech. 54, 39-48.

Westerweel, J. 1997 Fundamentals of digital particle image velocimetry. Meas. Sci. Technol. 8, 1379-1392.

White, F. M. 1974 Viscous Fluid Flow. McGraw-Hill.

WiEneke, B. 2008 Volume self-calibration for 3D particle image velocimetry. Exp. Fluids 45, $549-556$.

Williamson, C. H. K. 1996 Vortex dynamics in the cylinder wake. Annu. Rev. Fluid Mech. 28, 477-539.

Wygnanski, I., Champagne, F. \& Marsali, B. 1986 On the large-scale structures in two-dimensional, small-deficit, turbulent wakes. J. Fluid Mech. 168, 31-71.

Zhou, J., Adrian, R. J., Balachandra, S. \& Kendall, T. M. 1999 Mechanisms for generating coherent packets of hairpin vortices in channel flow. J. Fluid Mech. 387, 353-396. 